\title{
A drift homogenization problem revisited
}

\author{
MARC BRIANE AND PATRICK GÉRARD
}

\begin{abstract}
This paper revisits a homogenization problem studied by L. Tartar related to a tridimensional Stokes equation perturbed by a drift (related to the Coriolis force). Here, a scalar equation and a two-dimensional Stokes equation with a $L^{2}$-bounded oscillating drift are considered. Under higher integrability conditions the Tartar approach based on the oscillations test functions method applies and leads to a limit equation with an extra zero-order term. When the drift is only assumed to be equi-integrable in $L^{2}$, the same limit behaviour is obtained. However, the lack of integrability makes difficult the direct use of the Tartar method. A new method in the context of homogenization theory is proposed. It is based on a parametrix of the Laplace operator which permits to write the solution of the equation as a solution of a fixed point problem, and to use truncated functions even in the vector-valued case. On the other hand, two counter-examples which induce different homogenized zero-order terms actually show the sharpness of the equi-integrability assumption.
\end{abstract}

Mathematics Subject Classification (2010): 35B27 (primary); 76M50 (secondary).

\section{Introduction}

At the end of the Seventies L. Tartar developed his method based on oscillating test functions to deal with the homogenization of PDE's. In the particular framework of hydrodynamics $[14,15]$ he studied the Stokes equation in a bounded domain $\Omega$ of $\mathbb{R}^{3}$, perturbed by an oscillating drift term, i.e.

$$
\begin{cases}-\Delta u_{\varepsilon}+\operatorname{curl}\left(v_{\varepsilon}\right) \times u_{\varepsilon}+\nabla p_{\varepsilon}=f & \text { in } \Omega \\ \operatorname{div}\left(u_{\varepsilon}\right)=0 & \text { in } \Omega \\ u_{\varepsilon}=0 & \text { on } \partial \Omega\end{cases}
$$

where the oscillations are produced by the sequence of vector-valued functions $v_{\varepsilon}$ which weakly converges to some $v$ in $L^{3}(\Omega)^{3}$. L. Tartar proved that the limit equa-

Received June 16, 2010; accepted in revised form October 4, 2010. 
tion of (1.1) is the Brinkman [5] type equation

$$
\begin{cases}-\Delta u+\operatorname{curl}(v) \times u+\nabla p+M u=f & \text { in } \Omega \\ \operatorname{div}(u)=0 & \text { in } \Omega \\ u=0 & \text { on } \partial \Omega\end{cases}
$$

where $M$ is a positive definite symmetric matrix-valued function. More precisely, $M$ is defined by the convergences

$$
\left(D w_{\varepsilon}^{\lambda}\right)^{T} v_{\varepsilon} \longrightarrow M \lambda \quad \text { weakly in } L^{\frac{3}{2}}(\Omega)^{3}, \quad \text { for any } \lambda \in \mathbb{R}^{3},
$$

where $w_{\varepsilon}^{\lambda} \in W^{1,3}(\Omega)^{3}$ solves the Stokes equation (1.1) in which the term curl $\left(v_{\varepsilon}\right) \times$ $u_{\varepsilon}$ is replaced by $\operatorname{curl}\left(v_{\varepsilon}\right) \times \lambda$. Then, the convergence (1.3) combined with the compactness of $u_{\varepsilon}$ in $L^{3}(\Omega)^{3}$, yields the zero-order term $M u$ in (1.2). In [16] L. Tartar revisited this problem using the H-measures tool. On the other hand, the appearance of such a strange zero-order term in homogenization was also obtained from finely perforated domains by D. Cioranescu, F. Murat [6] for the Laplace equation, and by G. Allaire [2] for the Stokes equation, with zero Dirichlet boundary condition on the holes.

Since $\operatorname{curl}\left(v_{\varepsilon}\right) \times u_{\varepsilon}$ is orthogonal to $u_{\varepsilon}$, the energy associated with (1.1) is reduced to

$$
\int_{\Omega}\left|D u_{\varepsilon}\right|^{2} d x
$$

and thus does not depend on the drift $v_{\varepsilon}$. Starting from this remark our aim is to study two drift homogenization problems associated with the same energy (1.4), and to specify the best integrability condition satisfied by the drift so that the Tartar approach holds. The first problem is scalar and the second problem is a twodimensional equivalent of the Stokes problem (1.1). However, we have not succeeded in obtaining a similar result for the three-dimensional Stokes equation (1.1) since the best integrability assumption for $v_{\varepsilon}$ is not clear. $\Omega$ of $\mathbb{R}^{N}$,

In Section 2, we consider the following scalar equation in a bounded open set

$$
\begin{cases}-\Delta u_{\varepsilon}+b_{\varepsilon} \cdot \nabla u_{\varepsilon}+\operatorname{div}\left(b_{\varepsilon} u_{\varepsilon}\right)=f & \text { in } \Omega \\ u_{\varepsilon}=0 & \text { on } \partial \Omega,\end{cases}
$$

where $b_{\varepsilon} \in L^{\infty}(\Omega)^{N}$ is bounded in $L^{2}(\Omega)^{N}$. We obtain three different homogenization results:

In Section 2.1, assuming that the divergence of the drift $b_{\varepsilon}$ is bounded in $W^{-1, q}(\Omega)$, with $q>N$, we prove (see Theorem 2.1 ) that the sequence $u_{\varepsilon}$ weakly converges in $H_{0}^{1}(\Omega)$ to the solution $u$ of the equation

$$
\begin{cases}-\Delta u+b \cdot \nabla u+\operatorname{div}(b u)+\mu u=f & \text { in } \Omega \\ u=0 & \text { on } \partial \Omega,\end{cases}
$$


where $\mu$ is a nonnegative function. The proof follows the Tartar method using the oscillating test function

$$
w_{\varepsilon}:=\Delta^{-1}\left(\operatorname{div}\left(b_{\varepsilon}\right)\right) \in H_{0}^{1}(\Omega) .
$$

Then, in Section 2.2, assuming only the equi-integrability of the sequence $\nabla w_{\varepsilon}$ in $L^{2}(\Omega)^{N}$ (this is actually a weaker assumption than the equi-integrability of the whole sequence $b_{\varepsilon}$ ), we obtain (see Theorem 3.1) the limit problem (1.6) with

$$
\left|\nabla w_{\varepsilon}-\nabla w\right|^{2} \longrightarrow \mu \text { weakly in } L^{1}(\Omega) \text { and } \mu u^{2} \in L^{1}(\Omega) .
$$

It seems intricate to apply directly the Tartar method with the test function $w_{\varepsilon}$, since we cannot control the terms $b_{\varepsilon} \cdot \nabla u_{\varepsilon} w_{\varepsilon}$ and $b_{\varepsilon} \cdot \nabla w_{\varepsilon} u_{\varepsilon}$. To this end, one should consider truncations of both $w_{\varepsilon}$ and $\nabla w_{\varepsilon}$. To overcome this difficulty we propose a new method, up to our knowledge, in the context of homogenization theory, based on a parametrix of the Laplace operator. It follows that $u_{\varepsilon}$ reads as a solution of a fixed point problem, which allows us to estimate the sequence $\nabla w_{\varepsilon} \cdot \nabla u_{\varepsilon}$ only using a truncation of $\nabla w_{\varepsilon}$. The equi-integrability of $\nabla w_{\varepsilon}$ then gives the thesis. Also assuming that $b \in L^{q}(\Omega)^{N}$, with $q>N$, (which ensures the uniqueness in (1.6)) we prove the following corrector result

$$
u_{\varepsilon}-\left(1+w_{\varepsilon}-w\right) u \longrightarrow 0 \quad \text { strongly in } W_{\text {loc }}^{1, q}(\Omega), \quad \text { for any } q \in\left[1, N^{\prime}\right) .
$$

Finally, in Section 2.3, we show the sharpness of the equi-integrability condition thanks to a counter-example in the periodic framework (see Theorem 2.6). Making a change of functions with $b_{\varepsilon}=\nabla w_{\varepsilon}$, equation (1.5) is shown to be equivalent to the following equation

$$
-\Delta v_{\varepsilon}+\mu_{\varepsilon} v_{\varepsilon}=f_{\varepsilon}, \quad \text { with } \quad \mu_{\varepsilon}:=\left|\nabla w_{\varepsilon}\right|^{2},
$$

the solution of which has the same limit as $u_{\varepsilon}$. G. Dal Maso, A. Garroni [7] proved that the class of equations of type (1.10) is stable under homogenization. Here, we do not use this general result, but we explicit an oscillating sequence $w_{\varepsilon}$ so that the limit equation of (1.5), or equivalently (1.10), is

$$
-\Delta u+\gamma u=f
$$

with an explicit constant $\gamma$ which turns out to be $<\mu$. Therefore, the loss of equi-integrability for $\nabla w_{\varepsilon}$ violates the result of Section 2.2. Note that the vectorial character of the drift term in equation (1.5) makes difficult the derivation of a closure result similar to the one of [7] which is strongly based on the maximum principle.

In Section 3, we consider the following two-dimensional equivalent of the perturbed Stokes problem (1.1),

$$
\begin{cases}-\Delta u_{\varepsilon}+\operatorname{curl}\left(v_{\varepsilon}\right) J u_{\varepsilon}+\nabla p_{\varepsilon}=f & \text { in } \Omega \\ \operatorname{div}\left(u_{\varepsilon}\right)=0 & \text { in } \Omega \\ u_{\varepsilon}=0 & \text { on } \partial \Omega,\end{cases}
$$


where $J$ is the rotation matrix of angle $90^{\circ}$, and $v_{\varepsilon} \in L^{\infty}(\Omega)^{2}$ is bounded in $L^{2}(\Omega)^{2}$. Note that the weak formulation (3.4) of curl $\left(v_{\varepsilon}\right) J u_{\varepsilon}$ contains the drift term $\left(D u_{\varepsilon}\right)^{T} v_{\varepsilon}$, so that equation (1.12) can be also regarded as a drift problem. We follow the same scheme as in the scalar case:

In Section 3.1, assuming that the sequence $v_{\varepsilon}$ is bounded in $L^{r}(\Omega)^{2}$ with $r>2$, we show (see Theorem 3.1) that the sequence $u_{\varepsilon}$ weakly converges in $H_{0}^{1}(\Omega)$ to the solution $u$ of the Brinkman equation

$$
\begin{cases}-\Delta u+\operatorname{curl}(v) J u+\nabla p+M u=f & \text { in } \Omega \\ \operatorname{div}(u)=0 & \text { in } \Omega \\ u=0 & \text { on } \partial \Omega,\end{cases}
$$

where $M$ is a symmetric positive definite matrix-valued function defined by the convergence (1.3) in $L^{\frac{2 r}{r+2}}(\Omega)^{2}$.

In Section 3.2, assuming only the equi-integrability of the sequence $v_{\varepsilon}$ in $L^{2}(\Omega)^{2}$, we prove (see Theorem 3.3) owing to the Tartar method that the sequence $u_{\varepsilon}$ weakly converges in $H_{0}^{1}(\Omega)$ to the solution $u$ of the Brinkman equation (1.13) with similarly to (1.8),

$$
\left(D w_{\varepsilon}^{\lambda}\right)^{T} v_{\varepsilon} \longrightarrow M \lambda \quad \text { weakly in } L^{1}(\Omega)^{2} \text { and } M u \cdot u \in L^{1}(\Omega) .
$$

The proof is based on a double parametrix method carrying on both the velocity $u_{\varepsilon}$ and the pressure $p_{\varepsilon}$. However, the proof of the last estimate of (1.14) is more delicate than the one of (1.8), since we cannot use a comparison principle as in the scalar case. We need to introduce a test function similar to $w_{\varepsilon}^{\lambda}$ but associated with a truncation of $v_{\varepsilon}$. Moreover, if $\Omega$ has a regular boundary, $v \in L^{r}(\Omega)^{2}$ with $r>2$, and $M \in L^{m}(\Omega)^{2 \times 2}$ with $m>1$, we get the corrector result

$$
u_{\varepsilon}-u-W_{\varepsilon} u \longrightarrow 0 \text { strongly in } W^{1,1}(\Omega)^{2} \text {, where } W_{\varepsilon} \lambda:=w_{\varepsilon}^{\lambda} \text {, for } \lambda \in \mathbb{R}^{2} \text {. }
$$

Finally, in Section 3.2, we construct an oscillating sequence $v_{\varepsilon}$ which is not equiintegrable in $L^{2}(\Omega)^{2}$, which leads to the limit problem (1.13) involving a matrix $\Gamma$ which is not symmetric and satisfies the strict inequality

$$
\Gamma \lambda \cdot \lambda<M \lambda \cdot \lambda, \quad \text { for any } \lambda \neq 0,
$$

which is inconsistent with the Tartar approach. This shows the sharpness of the equi-integrability condition as in the scalar case. It would be very interesting to find the closure of the family of problems (1.12) under the sole condition of $L^{2}$ boundedness of the sequences $v_{\varepsilon}$. This problem is far from being evident due to the absence of comparison principle for such a vector-valued equation.

\section{Notations}

- The space dimension is $N \geq 2$, and $2^{*}:=\frac{2 N}{N-2}$. 
- The conjugate exponent of $p \geq 1$ is denoted by $p^{\prime}:=\frac{p}{p-1}$.

- For $u: \mathbb{R}^{N} \longrightarrow \mathbb{R}^{N}, D u:=\left(\frac{\partial u_{i}}{\partial x_{j}}\right)_{1 \leq i, j \leq N}$.

- For $\Sigma: \mathbb{R}^{N} \longrightarrow \mathbb{R}^{N \times N}, \operatorname{Div}(\Sigma):=\left(\sum_{j=1}^{N} \frac{\partial \Sigma_{i j}}{\partial x_{j}}\right)_{1 \leq i \leq N}$.

- $H_{\sharp}^{1}(Y)$, with $Y:=(0,1)^{N}$, denotes the space of the $Y$-periodic functions on $\mathbb{R}^{N}$ which belong to $H_{\text {loc }}^{1}\left(\mathbb{R}^{N}\right)$.

\section{A scalar equation with a drift term}

Along this section $\Omega$ is a bounded regular open set of $\mathbb{R}^{N}$, with $N \geq 2$, and $f$ is a distribution in $H^{-1}(\Omega)$.

\subsection{The classical case}

Let $q \in(N, \infty)$. Consider a sequence $b_{\varepsilon}$ in $L^{\infty}(\Omega)^{N}$ such that

$$
b_{\varepsilon} \longrightarrow b \text { weakly in } L^{2}(\Omega)^{N} \text { and } \operatorname{div}\left(b_{\varepsilon}\right) \text { is bounded in } W^{-1, q}(\Omega) .
$$

Let $w_{\varepsilon} \in W_{0}^{1, q}(\Omega)$ be the solution of the equation (see, e.g., [9, Theorem 2.1])

$$
\Delta w_{\varepsilon}=\operatorname{div}\left(b_{\varepsilon}\right) \quad \text { in } \mathcal{D}^{\prime}(\Omega)
$$

Up to a subsequence $w_{\varepsilon}$ weakly converges in $W_{0}^{1, q}(\Omega)$ to the function $w$ solution of

$$
\Delta w=\operatorname{div}(b) \quad \text { in } \mathcal{D}^{\prime}(\Omega) .
$$

We have the following result:

Theorem 2.1. The solution $u_{\varepsilon} \in H_{0}^{1}(\Omega)$ of the equation

$$
-\Delta u_{\varepsilon}+b_{\varepsilon} \cdot \nabla u_{\varepsilon}+\operatorname{div}\left(b_{\varepsilon} u_{\varepsilon}\right)=f \quad \text { in } \mathcal{D}^{\prime}(\Omega),
$$

weakly converges in $H_{0}^{1}(\Omega)$, up to a subsequence, to a solution $u \in H_{0}^{1}(\Omega)$ of the equation

$$
-\Delta u+b \cdot \nabla u+\operatorname{div}(b u)+\mu u=f \quad \text { in } \mathcal{D}^{\prime}(\Omega),
$$

where $\mu$ is the function defined by the convergence

$$
\left|\nabla w_{\varepsilon}-\nabla w\right|^{2} \longrightarrow \mu \text { weakly in } L^{\frac{q}{2}}(\Omega) .
$$

Remark 2.2. The uniqueness for equation (2.4) is not evident under the sole assumption $b \in L^{2}(\Omega)^{2}$. Assuming a stronger integrability of $b$ we will obtain in Theorem 2.4 the uniqueness for the limit equation. 
Proof. The proof is based on the choice of appropriate oscillating test functions as Tartar did (see [13, Appendix], and [17]). The function $w_{\varepsilon}$ of (2.2) will play the role of the oscillating test function. The variational formulation of (2.4) is

$$
\begin{aligned}
\int_{\Omega} \nabla u_{\varepsilon} \cdot \nabla \varphi d x & +\int_{\Omega} b_{\varepsilon} \cdot \nabla u_{\varepsilon} \varphi d x-\int_{\Omega} b_{\varepsilon} \cdot \nabla \varphi u_{\varepsilon} d x \\
& =\langle f, \varphi\rangle_{H^{-1}(\Omega), H_{0}^{1}(\Omega)}, \quad \forall \varphi \in H_{0}^{1}(\Omega) .
\end{aligned}
$$

Then, by the Lax-Milgram theorem there exists a unique solution $u_{\varepsilon}$ of (2.7) in $H_{0}^{1}(\Omega)$. In particular, for $v \in W^{1, \infty}(\Omega)$, putting $\varphi=v u_{\varepsilon}$ as test function in (2.7) we obtain the identity

$$
\int_{\Omega}\left|\nabla u_{\varepsilon}\right|^{2} v d x+\int_{\Omega} \nabla u_{\varepsilon} \cdot \nabla v u_{\varepsilon} d x-\int_{\Omega} b_{\varepsilon} \cdot \nabla v u_{\varepsilon}^{2} d x=\left\langle f, v u_{\varepsilon}\right\rangle_{H^{-1}(\Omega), H_{0}^{1}(\Omega)}
$$

which will be used several times. So, choosing $v=1$ in (2.8) the term with $b_{\varepsilon}$ cancel so that we easily deduce that $u_{\varepsilon}$ is bounded in $H_{0}^{1}(\Omega)$ and weakly converges, up to a subsequence, to a function $u$ in $H_{0}^{1}(\Omega)$. Therefore, it follows from (2.7) the limit variational formulation

$$
\int_{\Omega} \nabla u \cdot \nabla \varphi d x+\int_{\Omega} b \cdot \nabla u \varphi d x+\int_{\Omega} \varphi d v-\int_{\Omega} b \cdot \nabla \varphi u d x=\langle f, \varphi\rangle_{H^{-1}(\Omega), H_{0}^{1}(\Omega)}
$$

which holds for any $\varphi \in W_{0}^{1, q}(\Omega)$ (due to the embedding of $W_{0}^{1, q}(\Omega)$ into $C(\bar{\Omega})$ for $q>N$ ), where the measure $v$ is defined by the convergence

$$
b_{\varepsilon} \cdot \nabla u_{\varepsilon} \longrightarrow b \cdot \nabla u+v \quad \text { weakly-* in } \mathcal{M}(\Omega) .
$$

The limit equation associated with (2.9) is

$$
-\Delta u+b \cdot \nabla u+v+\operatorname{div}(b u)=f \quad \text { in } \mathcal{D}^{\prime}(\Omega)
$$

Now, let us determine the measure $v$ of (2.10). Let $\varphi \in C_{c}^{\infty}(\Omega)$. Putting $\varphi w_{\varepsilon}$ as test function in (2.7) and $\varphi u_{\varepsilon}$ in (2.2), and taking the difference of the two equalities we get

$$
\begin{aligned}
& \int_{\Omega} \nabla u_{\varepsilon} \cdot \nabla \varphi w_{\varepsilon} d x-\int_{\Omega} \nabla w_{\varepsilon} \cdot \nabla \varphi u_{\varepsilon} d x \\
& =\left\langle f, \varphi w_{\varepsilon}\right\rangle_{H^{-1}(\Omega), H_{0}^{1}(\Omega)}-\int_{\Omega} b_{\varepsilon} \cdot \nabla u_{\varepsilon} \varphi w_{\varepsilon} d x+\int_{\Omega} b_{\varepsilon} \cdot \nabla w_{\varepsilon} \varphi u_{\varepsilon} d x \\
& \quad+\int_{\Omega} b_{\varepsilon} \cdot \nabla \varphi u_{\varepsilon} w_{\varepsilon} d x-\int_{\Omega} b_{\varepsilon} \cdot \nabla u_{\varepsilon} \varphi d x-\int_{\Omega} b_{\varepsilon} \cdot \nabla \varphi u_{\varepsilon} d x
\end{aligned}
$$


Passing to the limit in (2.12) by using the strong convergence of $u_{\varepsilon}$ in $L^{p}(\Omega)$, for $p<2^{*}$, and the uniform convergence of $w_{\varepsilon}$ in $C(\bar{\Omega})(q>N)$, we obtain

$$
\begin{aligned}
& \int_{\Omega} \nabla u \cdot \nabla \varphi w d x-\int_{\Omega} \nabla w \cdot \nabla \varphi u d x \\
& =\langle f, \varphi w\rangle_{H^{-1}(\Omega), H_{0}^{1}(\Omega)}-\int_{\Omega} b \cdot \nabla u \varphi w d x-\int_{\Omega} \varphi w d v+\int_{\Omega} \sigma \varphi u d x \\
& \quad+\int_{\Omega} b \cdot \nabla \varphi u w d x-\int_{\Omega} b \cdot \nabla u \varphi d x-\int_{\Omega} \varphi d v-\int_{\Omega} b \cdot \nabla \varphi u d x
\end{aligned}
$$

where the measure $v$ is defined by (2.10) and the function $\sigma$ is defined, up to a subsequence, by the convergence

$$
b_{\varepsilon} \cdot \nabla w_{\varepsilon} \longrightarrow \sigma \quad \text { weakly in } L^{\frac{2 q}{q+2}}(\Omega) .
$$

On the other hand, putting $\varphi w \in W_{0}^{1, q}(\Omega)$ in (2.9) and $\varphi u \in H_{0}^{1}(\Omega)$ in (2.3) we have

$$
\begin{aligned}
\int_{\Omega} \nabla u \cdot \nabla(\varphi w) d x= & \langle f, \varphi w\rangle_{H^{-1}(\Omega), H_{0}^{1}(\Omega)}-\int_{\Omega} b \cdot \nabla u \varphi w d x-\int_{\Omega} \varphi w d v \\
& +\int_{\Omega} b \cdot \nabla w \varphi u d x+\int_{\Omega} b \cdot \nabla \varphi u w d x, \\
\int_{\Omega} \nabla w \cdot \nabla(\varphi u) d x= & \int_{\Omega} b \cdot \nabla u \varphi d x+\int_{\Omega} b \cdot \nabla \varphi u d x .
\end{aligned}
$$

Equating the difference between (2.15) and (2.16) to the right-hand side of (2.13), it follows that

$$
\int_{\Omega} \sigma \varphi u d x-\int_{\Omega} b \cdot \nabla w \varphi u d x-\int_{\Omega} \varphi d v=0, \quad \text { for any } \varphi \in C_{c}^{\infty}(\Omega),
$$

which implies that

$$
v=\sigma u-b \cdot \nabla w u \quad \text { in } \mathcal{D}^{\prime}(\Omega)
$$

It thus remains to determine the limit equation (2.5). To this end, we pass to the limit by using $\varphi w_{\varepsilon}$ as test function in (2.2) and the definition (2.6) of $\mu$, and we put $\varphi w$ in (2.3), which yields

$$
\begin{aligned}
& \int_{\Omega}\left(\mu+|\nabla w|^{2}\right) \varphi d x+\int_{\Omega} \nabla w \cdot \nabla \varphi w d x=\int_{\Omega} \sigma \varphi d x+\int_{\Omega} b \cdot \nabla \varphi w d x, \\
& \int_{\Omega}|\nabla w|^{2} \varphi d x+\int_{\Omega} \nabla w \cdot \nabla \varphi w d x=\int_{\Omega} b \cdot \nabla w \varphi d x+\int_{\Omega} b \cdot \nabla \varphi w d x .
\end{aligned}
$$

Equating (2.19) and (2.20), we deduce that

$$
\mu=\sigma-b \cdot \nabla w \quad \text { in } \mathcal{D}^{\prime}(\Omega),
$$


which combined with (2.18) implies that

$$
v=\mu u \text { in } \mathcal{D}^{\prime}(\Omega) \text {. }
$$

Finally, the limit equation (2.11) and the relation (2.22) give the desired homogenized equation (2.5).

Remark 2.3. It can be shown that

$$
\mu(x)=\int_{S^{N-1}} \boldsymbol{\mu}(x, d \xi) \xi \cdot \xi,
$$

where $\boldsymbol{\mu}$ denotes the matrix-valued $H$-measure (or micro-local defect measure) of the sequence $b_{\varepsilon}$ (see [16] and [8]), and $S^{N-1}$ the unit sphere of $\mathbb{R}^{N}$.

Assumption (2.1) is actually not sharp. In the next section we replace it by the boundedness of $b_{\varepsilon}$ and the equi-integrability of $\nabla w_{\varepsilon}$ in $L^{2}(\Omega)^{2}$.

\subsection{The case under an equi-integrability assumption}

In this section $\Omega$ is a bounded open set of $\mathbb{R}^{N}$. Consider a sequence $b_{\varepsilon}$ in $L^{\infty}(\Omega)^{N}$ the Hodge decomposition of which is

$$
b_{\varepsilon}=\nabla w_{\varepsilon}+\xi_{\varepsilon}, \quad \text { with } \quad w_{\varepsilon} \in H_{0}^{1}(\Omega), \xi_{\varepsilon} \in L^{2}(\Omega)^{N} \text { and } \operatorname{div}\left(\xi_{\varepsilon}\right)=0,
$$

such that

$$
b_{\varepsilon} \longrightarrow b \text { weakly in } L^{2}(\Omega)^{N} \text {. }
$$

Note that for a fixed $\varepsilon>0, w_{\varepsilon} \in W^{1, p}(\Omega)$ and $\xi_{\varepsilon} \in L^{p}(\Omega)^{N}$ for any $p \in[2, \infty)$. But the essential point is the asymptotic behaviour of the sequences $b_{\varepsilon}, \nabla w_{\varepsilon}, \xi_{\varepsilon}$. Our main assumption is the equi-integrability of the sequence $\nabla w_{\varepsilon}$ in $L^{2}(\Omega)^{N}$. By virtue of the Vitali-Saks theorem this is equivalent to the following convergence, up to an extraction of a subsequence,

$$
\left|\nabla w_{\varepsilon}-\nabla w\right|^{2} \longrightarrow \mu \text { weakly in } L^{1}(\Omega),
$$

(Compare to (2.6) with $q>N$ ).

We have the following result:

\section{Theorem 2.4.}

i) Under the equi-integrability assumption (2.26) the solution $u_{\varepsilon}$ of (2.4) weakly converges in $H_{0}^{1}(\Omega)$ to a solution $u$ of the equation

$$
-\Delta u+b \cdot \nabla u+\operatorname{div}(b u)+\mu u=f \quad \text { in } \mathcal{D}^{\prime}(\Omega),
$$

with

$$
\int_{\Omega} \mu u^{2} d x \leq\langle f, u\rangle_{H^{-1}(\Omega), H_{0}^{1}(\Omega)}-\int_{\Omega}|\nabla u|^{2} d x .
$$


ii) Also assume that $b \in L^{q}(\Omega)^{N}$, where $q>2$ if $N=2$ and $q=N$ if $N>2$. Then, we have

$$
\int_{\Omega}|\nabla u|^{2} d x+\int_{\Omega} \mu u^{2} d x=\langle f, u\rangle_{H^{-1}(\Omega), H_{0}^{1}(\Omega)},
$$

and there exists a unique solution $u \in H_{0}^{1}(\Omega)$ of equation (2.27), with $\mu u^{2} \in$ $L^{1}(\Omega)$.

Moreover, for any $p \in[1,2)$ if $N=2$ and $p=N^{\prime}$ if $N>2$, we have the corrector result

$$
\nabla u_{\varepsilon}-\nabla u-\left(\nabla w_{\varepsilon}-\nabla w\right) u \longrightarrow 0 \quad \text { strongly in } L_{\mathrm{loc}}^{p}(\Omega)^{N},
$$

and for any $r \in[1, p)$,

$$
u_{\varepsilon}-\left(1+w_{\varepsilon}-w\right) u \longrightarrow 0 \text { strongly in } W_{\mathrm{loc}}^{1, r}(\Omega) .
$$

Remark 2.5. No equi-integrability is required for the divergence free sequence $\xi_{\varepsilon}$. Actually, we can prove that the equi-integrability of the sequence $b_{\varepsilon}$ in $L^{2}(\Omega)^{N}$ implies the equi-integrability of its two components $\nabla w_{\varepsilon}, \xi_{\varepsilon}$ in $L_{\mathrm{loc}}^{2}(\Omega)^{N}$. Therefore, condition (2.26) is really weaker than the equi-integrability of $b_{\varepsilon}$.

Moreover, the equi-integrability of $\nabla w_{\varepsilon}$ in $L^{2}(\Omega)^{N}$ is essential for deriving the limit equation with the zero-order term $\mu u$. When this condition is not satisfied we can obtain a similar limit equation but with a different zero-order term (see Section 2.3).

Proof of Theorem 2.4. The limit $u$ of $u_{\varepsilon}$ in $H_{0}^{1}(\Omega)$ solves the equation (2.11) where $v$ is defined by

$$
b_{\varepsilon} \cdot \nabla u_{\varepsilon}-b \cdot \nabla u \longrightarrow v \quad \text { weakly-* in } \mathcal{M}(\Omega)
$$

We thus have

$$
b_{\varepsilon} \cdot \nabla u_{\varepsilon}=\left(\xi_{\varepsilon}+\nabla w\right) \cdot \nabla u_{\varepsilon}+\left(\nabla w_{\varepsilon}-\nabla w\right) \cdot \nabla u_{\varepsilon} \longrightarrow b \cdot \nabla u+v \quad \text { in } \mathcal{D}^{\prime}(\Omega) .
$$

Moreover, by the Murat, Tartar div-curl lemma [11] the sequence $\left(\xi_{\varepsilon}+\nabla w\right) \cdot \nabla u_{\varepsilon}$ converges to $(\xi+\nabla w) \cdot \nabla u=b \cdot \nabla u$. This combined with the equi-integrability of $\nabla w_{\varepsilon}$ implies that $v$ is also given by the convergence

$$
\left(\nabla w_{\varepsilon}-\nabla w\right) \cdot \nabla u_{\varepsilon} \longrightarrow v \quad \text { weakly in } L^{1}(\Omega) \text {. }
$$

The proof of Theorem 2.4. is based on a parametrix method which allows us to express $u_{\varepsilon}$ as a solution of a fixed point problem. As a consequence, we obtain a strong estimate of $\nabla u_{\varepsilon}$ in $L_{\text {loc }}^{p}(\Omega)$ for some $p>1$ close to 1 . However, this estimate cannot provide directly the desired limit $v$ of (2.33) since $p<2$. To overcome this difficulty we consider a truncation $\eta_{\varepsilon}^{k}$ of $\nabla w_{\varepsilon}$ which is bounded by $k>0$. Then, we 
can pass to the limit as $\varepsilon$ tends to zero in the product $\eta_{\varepsilon}^{k} \cdot \nabla u_{\varepsilon}$ for a fixed $k$. Hence, thanks to the equi-integrability of $\nabla w_{\varepsilon}$ we deduce the limit $v$ as $k$ tends to infinity.

The proof is divided into four steps. In the first step we present the parametrix method which leads to a $L^{p}$-strong estimate of $\nabla u_{\varepsilon}$. In the second step we determine the limit of the sequence $\eta_{\varepsilon}^{k} \cdot \nabla u_{\varepsilon}$ for a fixed $k>0$. In the third step we determine the limit $v$ and the limit equation (2.27) together with (2.28). The fourth step is devoted to the proof of equality (2.29) and the corrector results (2.30) and (2.31).

First step. The parametrix method.

First, let us define a parametrix for the Laplace operator in $\Omega$. To this end consider two sequences of functions $\varphi_{n}, \psi_{n}$ in $C_{c}^{\infty}(\Omega)$, such that

$$
\left\{\begin{array}{l}
0 \leq \varphi_{n}, \psi_{n} \leq 1 \text { and } \varphi_{n}=1 \text { in } \operatorname{supp}\left(\psi_{n}\right), \text { for any } n \geq 1, \\
\left\{n \geq 1: \operatorname{supp}\left(\psi_{n}\right) \cap K \neq \varnothing\right\} \text { is finite, for any compact subset } K \subset \Omega, \\
\sum_{n \geq 1} \psi_{n}=1 \text { in } \Omega .
\end{array}\right.
$$

Let $E$ be the fundamental solution of the Laplace operator in $\mathbb{R}^{N}$. Then, the operator $P$ defined in $\mathcal{D}^{\prime}(\Omega)$ by

$$
P(\zeta):=\sum_{n \geq 1} \psi_{n} E *\left(\varphi_{n} \zeta\right), \quad \text { for } \zeta \in \mathcal{D}^{\prime}(\Omega),
$$

is a parametrix of the Laplace operator (see [1, Chapter I], for further details) which satisfies

$$
P(\Delta \zeta)=\zeta-K(\zeta) \quad \text { and } \quad \Delta(P(\zeta))=\zeta-K^{\prime}(\zeta), \quad \text { for } \zeta \in \mathcal{D}^{\prime}(\Omega),
$$

where $K, K^{\prime}$ are two $C^{\infty}$-kernel operators properly supported in $\Omega$. Thanks to the Calderòn-Zygmund regularity for the Laplace operator (see, e.g., [9, Theorem 2.1], and the references therein) we also have for any $p>1$, and $s \in[0,2]$ such that $s+\frac{1}{p}$ is not an integer,

$$
P \text { maps continuously } \mathcal{D}^{\prime}(\Omega) \text { to } \mathcal{D}^{\prime}(\Omega) \text {, and } W_{\text {loc }}^{-s, p}(\Omega) \text { to } W_{\mathrm{loc}}^{2-s, p}(\Omega) \text {. }
$$

Then, applying (2.36) to the solution $u_{\varepsilon}$ of (2.4) we have

$$
\begin{aligned}
u_{\varepsilon}= & P\left(\Delta u_{\varepsilon}\right)+K\left(u_{\varepsilon}\right) \\
= & P\left(\operatorname{div}\left[u\left(\nabla w_{\varepsilon}-\nabla w\right)\right]\right)+P\left(\operatorname{div}\left[\nabla w_{\varepsilon}\left(u_{\varepsilon}-u\right)\right]\right) \\
& +P(\operatorname{div}(u \nabla w))+P\left(\xi_{\varepsilon} \cdot \nabla u_{\varepsilon}+b_{\varepsilon} \cdot \nabla u_{\varepsilon}-f\right)+K\left(u_{\varepsilon}\right),
\end{aligned}
$$

Fix $p>1$ close enough to 1 and $s \in\left(N / p^{\prime}, 1\right)$. Since $u_{\varepsilon}-u$ strongly converges to 0 in $L^{q}(\Omega)$ for any $q \in\left(2,2^{*}\right)$, the sequence $\operatorname{div}\left(\nabla w_{\varepsilon}\left(u_{\varepsilon}-u\right)\right)$ strongly converges to 0 in $W^{-1, p}(\Omega)$, hence by $(2.37)$ we have

$$
P\left(\operatorname{div}\left[\nabla w_{\varepsilon}\left(u_{\varepsilon}-u\right)\right]\right) \longrightarrow 0 \quad \text { strongly in } W_{\text {loc }}^{1, p}(\Omega) .
$$


Moreover, the sequence $\xi_{\varepsilon} \cdot \nabla u_{\varepsilon}+b_{\varepsilon} \cdot \nabla u_{\varepsilon}$ is bounded in $L^{1}(\Omega)$, thus in $W^{-s, p}(\Omega)$ since $s>N / p^{\prime}$. Therefore, again by (2.37) the sequence $\nabla P\left(\xi_{\varepsilon} \cdot \nabla u_{\varepsilon}+b_{\varepsilon}\right.$. $\left.\nabla u_{\varepsilon}-f\right)$ is bounded in $W^{1-s, p}(\Omega)^{N}$, and up to a subsequence strongly converges in $L_{\text {loc }}^{p}(\Omega)^{N}$. Hence, since

$$
\xi_{\varepsilon} \cdot \nabla u_{\varepsilon}+b_{\varepsilon} \cdot \nabla u_{\varepsilon} \longrightarrow \xi \cdot \nabla u+v+b \cdot \nabla u \quad \text { in } \mathcal{D}^{\prime}(\Omega)
$$

we deduce from (2.38) the strong estimate

$$
\begin{aligned}
& \nabla u_{\varepsilon}-\nabla P\left(\operatorname{div}\left[u\left(\nabla w_{\varepsilon}-\nabla w\right)\right]\right) \\
& =\nabla P(\operatorname{div}(u \nabla w)+\xi \cdot \nabla u+v+b \cdot \nabla u-f)+\nabla K(u)+o_{L_{\mathrm{loc}}^{p}(\Omega)^{N}}(1) \\
& =\nabla P(v+b \cdot \nabla u+\operatorname{div}(b u)-f)+\nabla K(u)+o_{L_{\mathrm{loc}}^{p}(\Omega)^{N}}(1) \\
& \quad(\xi \cdot \nabla u=\operatorname{div}(u \xi)),
\end{aligned}
$$

where $o_{L_{\text {loc }}^{p}(\Omega)^{N}}(1)$ denotes a sequence which strongly converges to 0 in $L_{\text {loc }}^{p}(\Omega)^{N}$. On the other hand, by (2.36) and (2.37) we have

$$
\begin{aligned}
\nabla P\left(\operatorname{div}\left[u\left(\nabla w_{\varepsilon}-\nabla w\right)\right]\right) & =\nabla P\left(\Delta\left[u\left(w_{\varepsilon}-w\right)\right]\right)-\nabla P\left(\operatorname{div}\left[\nabla u\left(w_{\varepsilon}-w\right)\right]\right) \\
& =\nabla P\left(\Delta\left[u\left(w_{\varepsilon}-w\right)\right]\right)+o_{L_{\mathrm{loc}}^{p}(\Omega)^{N}}(1) \\
& =\nabla\left(u\left(w_{\varepsilon}-w\right)\right)+o_{L_{\mathrm{loc}}^{p}(\Omega)^{N}}(1) \\
& =u\left(\nabla w_{\varepsilon}-\nabla w\right)+o_{L_{\mathrm{loc}}^{p}(\Omega)^{N}}(1) .
\end{aligned}
$$

Therefore, this combined with (2.39) yields

$$
\begin{aligned}
\nabla u_{\varepsilon}-u\left(\nabla w_{\varepsilon}-\nabla w\right)= & \nabla P(v+b \cdot \nabla u+\operatorname{div}(b u)-f)+\nabla K(u) \\
& +o_{L_{\mathrm{loc}}^{p}(\Omega)^{N}}(1) .
\end{aligned}
$$

Second step. Estimate of the sequence $\eta_{\varepsilon}^{k} \cdot \nabla u_{\varepsilon}$.

Set $\eta_{\varepsilon}^{k}:=\nabla w_{\varepsilon} 1_{\left\{\left|\nabla w_{\varepsilon}\right|<k\right\}}$, for a positive integer $k$. Let us determine the limit of $\eta_{\varepsilon}^{k} \cdot \nabla u_{\varepsilon}$ in $L_{\text {loc }}^{2}(\Omega)^{N}$. Using a diagonal extraction, there exists a subsequence of $\varepsilon$, still denoted by $\varepsilon$, such that $\eta_{\varepsilon}^{k}$ weakly converges to some $\eta^{k}$ in $L^{\infty}(\Omega)^{N}$ for any $k$. By the strong convergence (2.40) combined with the weak convergence of $u\left(\nabla w_{\varepsilon}-\nabla w\right)$ to 0 in $L^{p}(\Omega)^{N}$ (for $p$ close to 1 ) we have

$$
\begin{aligned}
& \eta_{\varepsilon}^{k} \cdot \nabla u_{\varepsilon}-\left(\eta_{\varepsilon}^{k}-\eta^{k}\right) \cdot\left(\nabla w_{\varepsilon}-\nabla w\right) u \\
& \longrightarrow \eta^{k} \cdot \nabla P(v+b \cdot \nabla u+\operatorname{div}(b u)-f)+\eta^{k} \cdot \nabla K(u) \quad \text { weakly in } L_{\text {loc }}^{p}(\Omega) .
\end{aligned}
$$

Hence, we get that

$$
\sigma^{k}=\mu^{k} u+\eta^{k} \cdot \nabla P(v+b \cdot \nabla u+\operatorname{div}(b u)-f)+\eta^{k} \cdot \nabla K(u) \quad \text { in } \Omega,
$$


where

$$
\left\{\begin{aligned}
& \sigma^{k}:=\lim _{\varepsilon \rightarrow 0}\left[\eta_{\varepsilon}^{k} \cdot \nabla u_{\varepsilon}\right] \text { weakly in } L^{2}(\Omega), \\
& \mu^{k}:=\lim _{\varepsilon \rightarrow 0}\left[\left(\eta_{\varepsilon}^{k}-\eta^{k}\right) \cdot\left(\nabla w_{\varepsilon}-\nabla w\right)\right] \text { weakly in } L^{2}(\Omega) .
\end{aligned}\right.
$$

Third step. Determination of $v$ and the limit equation (2.27).

Starting from the limit equation (2.11) we have by (2.36)

$$
u=P(v+b \cdot \nabla u+\operatorname{div}(b u)-f)+K(u) \text { in } \Omega,
$$

hence

$$
\eta^{k} \cdot \nabla u=\eta^{k} \cdot \nabla P(v+b \cdot \nabla u+\operatorname{div}(b u)-f)+\eta^{k} \cdot \nabla K(u) \quad \text { in } \Omega .
$$

Equating this with (2.41) we obtain

$$
\sigma^{k}=\mu^{k} u+\eta^{k} \cdot \nabla u \quad \text { in } \Omega .
$$

Now, let us pass to the limit as $k \rightarrow \infty$. By virtue of the equi-integrability of $\nabla w_{\varepsilon}$ in $L^{2}(\Omega)^{N}$ and by definition (2.42) the sequence $\mu^{k}$ strongly converges in $L^{1}(\Omega)$ to the function $\mu$ of (2.26), $\eta^{k}$ strongly converges to $\nabla w$ in $L^{2}(\Omega)^{N}$, and $\sigma^{k}$ strongly converges to $v+\nabla w \cdot \nabla u$ in $L^{1}(\Omega)$. Then, up to a subsequence $\mu^{k}$ converges to $\mu$ a.e. in $\Omega$, and by the Fatou lemma combined with equality (2.43) we get

$$
\begin{aligned}
\int_{\Omega}|\mu u| d x & \leq \liminf _{k \rightarrow \infty} \int_{\Omega}\left|\mu^{k} u\right| d x \\
& \leq \liminf _{k \rightarrow \infty} \int_{\Omega}\left|\sigma^{k}-\eta^{k} \cdot \nabla u\right| d x=\int_{\Omega}|\nu| d x .
\end{aligned}
$$

We deduce from (2.44) and (2.43) that $\mu u \in L^{1}(\Omega)$ and

$$
v=\mu u \text { in } \Omega,
$$

which yields the limit equation (2.27).

It remains to prove the inequality of (2.28). Let $v \in L^{\infty}(\Omega)$ and $t \in \mathbb{R}$. By (2.26), (2.33) and (2.45) we have

$$
\begin{aligned}
& \left.\int_{\Omega} \mid \nabla u_{\varepsilon}-\nabla u-\left(\nabla w_{\varepsilon}-\nabla w\right) t v\right)\left.\right|^{2} d x \\
& =\int_{\Omega}\left|\nabla u_{\varepsilon}-\nabla u\right|^{2} d x+t^{2} \int_{\Omega}\left|\nabla w_{\varepsilon}-\nabla w\right|^{2} v^{2} d x \\
& \quad-2 t \int_{\Omega} \nabla u_{\varepsilon} \cdot\left(\nabla w_{\varepsilon}-\nabla w\right) v d x+o(1) \\
& =\langle f, u\rangle_{H^{-1}(\Omega), H_{0}^{1}(\Omega)}-\int_{\Omega}|\nabla u|^{2} d x+t^{2} \int_{\Omega} \mu v^{2} d x \\
& \quad-2 t \int_{\Omega} \mu u v d x+o(1),
\end{aligned}
$$


hence

$t^{2} \int_{\Omega} \mu v^{2} d x-2 t \int_{\Omega} \mu u v d x+\langle f, u\rangle_{H^{-1}(\Omega), H_{0}^{1}(\Omega)}-\int_{\Omega}|\nabla u|^{2} d x \geq 0, \quad \forall t \in \mathbb{R}$.

This implies that

$$
\left(\int_{\Omega} \mu u v d x\right)^{2} \leq\left(\langle f, u\rangle_{H^{-1}(\Omega), H_{0}^{1}(\Omega)}-\int_{\Omega}|\nabla u|^{2} d x\right) \int_{\Omega} \mu v^{2} d x .
$$

Let $T_{k}, k>0$, be a function in $C^{1}(\mathbb{R})$ such that

$$
0 \leq T_{k}^{\prime} \leq 1 \quad \text { and } \quad \begin{cases}T_{k}(t)=t & \text { if }|t| \leq k \\ \left|T_{k}(t)\right|=k+1 & \text { if }|t| \geq k+2\end{cases}
$$

Putting $v=T_{k}(u)$ as test function in (2.47) and using that $T_{k}(u)^{2} \leq u T_{k}(u)$, we get

$$
\left(\int_{\Omega} \mu u T_{k}(u) d x\right)^{2} \leq\left(\langle f, u\rangle_{H^{-1}(\Omega), H_{0}^{1}(\Omega)}-\int_{\Omega}|\nabla u|^{2} d x\right) \int_{\Omega} \mu u T_{k}(u) d x,
$$

hence

$$
\int_{\Omega} \mu u T_{k}(u) d x \leq\langle f, u\rangle_{H^{-1}(\Omega), H_{0}^{1}(\Omega)}-\int_{\Omega}|\nabla u|^{2} d x .
$$

Since $u T_{k}(u)$ is a nondecreasing nonnegative sequence which converges to $u^{2}$ a.e. in $\Omega$, the Beppo-Levi theorem applied to (2.49) thus gives inequality (2.28).

Fourth step. Proof of equality (2.29) and of the corrector results (2.30), (2.31).

Assume that $b \in L^{q}(\Omega)^{N}$, where $q>2$ if $N=2$ and $q=N$ if $N>2$. Let $\varphi_{n}$ be a sequence in $C_{0}^{1}(\mathbb{R})$ which strongly converges to $u$ in $H_{0}^{1}(\Omega)$ and a.e. in $\Omega$, and such that $\left|\nabla \varphi_{n}\right|$ is dominated by a fixed function in $L^{2}(\Omega)$. Putting the truncation function $T_{k}\left(\varphi_{n}\right)$ (2.48) in the limit equation (2.27) we have

$$
\begin{aligned}
& \int_{\Omega} \nabla u \cdot \nabla T_{k}\left(\varphi_{n}\right) d x+\int_{\Omega} b \cdot \nabla u T_{k}\left(\varphi_{n}\right) d x-\int_{\Omega} b \cdot \nabla T_{k}\left(\varphi_{n}\right) u d x \\
& \quad+\int_{\Omega} \mu u T_{k}\left(\varphi_{n}\right) d x \\
& =\left\langle f, T_{k}\left(\varphi_{n}\right)\right\rangle_{H^{-1}(\Omega), H_{0}^{1}(\Omega)} .
\end{aligned}
$$

Since $b \cdot \nabla u, \mu u \in L^{1}(\Omega)$ and $b u \in L^{2}(\Omega)^{N}$ (as a consequence of $b \in L^{q}(\Omega)^{N}$ ), we can pass to the limit as $n \rightarrow \infty$ in the previous equality owing to the Lebesgue dominated convergence theorem, which yields

$$
\begin{aligned}
& \int_{\Omega} \nabla u \cdot \nabla T_{k}(u) d x+\int_{\Omega} b \cdot \nabla u T_{k}(u) d x-\int_{\Omega} b \cdot \nabla T_{k}(u) u d x \\
& \quad+\int_{\Omega} \mu u T_{k}(u) d x \\
& =\left\langle f, T_{k}(u)\right\rangle_{H^{-1}(\Omega), H_{0}^{1}(\Omega)} .
\end{aligned}
$$


Then, using that $\left|T_{k}(u)\right| \leq|u|, 0 \leq T_{k}^{\prime}(u) \leq 1, T_{k}(u)$ strongly converges to $u$ in $H_{0}^{1}(\Omega)$, and that $b u \in L^{2}(\Omega)^{N}, \mu u^{2} \in L^{1}(\Omega)$, and passing to the limit as $k \rightarrow \infty$ owing to the Lebesgue dominated convergence theorem we get

$\int_{\Omega}|\nabla u|^{2} d x+\int_{\Omega} b \cdot \nabla u u d x-\int_{\Omega} b \cdot \nabla u u d x+\int_{\Omega} \mu u^{2} d x=\langle f, u\rangle_{H^{-1}(\Omega), H_{0}^{1}(\Omega)}$,

which is (2.29). Moreover, the proof of equality (2.29) with $f=0$ shows that there exists a unique solution $u \in H_{0}^{1}(\Omega)$ of equation (2.27), with $\mu u^{2} \in L^{1}(\Omega)$.

It remains to prove the corrector results. By the estimate (2.46) with $v=T_{k}(u)$ and $t=1$, combined with equality (2.29) we have

$$
\begin{aligned}
& \lim _{k \rightarrow \infty} \lim _{\varepsilon \rightarrow 0}\left(\int_{\Omega}\left|\nabla u_{\varepsilon}-\nabla u-\left(\nabla w_{\varepsilon}-\nabla w\right) T_{k}(u)\right|^{2} d x\right) \\
& =\lim _{k \rightarrow \infty}\left(\int_{\Omega} \mu\left(u-T_{k}(u)\right)^{2} d x\right)=0 .
\end{aligned}
$$

On the other hand, let $p \in[1,2)$ if $N=2$ and $p=N^{\prime}$ if $N>2$, and consider an open set $\omega \Subset \Omega$. By the Hölder inequality we have

$$
\begin{aligned}
& \int_{\omega}\left|\nabla u_{\varepsilon}-\nabla u-\left(\nabla w_{\varepsilon}-\nabla w\right) u\right|^{p} d x \\
& \leq 2^{p-1}\left(\int_{\Omega}\left|\nabla u_{\varepsilon}-\nabla u-\left(\nabla w_{\varepsilon}-\nabla w\right) T_{k}(u)\right|^{p}\right. \\
& \left.\quad+\int_{\omega}\left|\nabla w_{\varepsilon}-\nabla w\right|^{p}\left|u-T_{k}(u)\right|^{p} d x\right) \\
& \leq c\left(\int_{\Omega}\left|\nabla u_{\varepsilon}-\nabla u-\left(\nabla w_{\varepsilon}-\nabla w\right) T_{k}(u)\right|^{2}\right)^{\frac{p}{2}} \\
& \quad+c\left(\int_{\omega}\left|u-T_{k}(u)\right|^{\frac{2 p}{2-p}} d x\right)^{1-\frac{p}{2}} \\
& \leq c\left(\int_{\Omega}\left|\nabla u_{\varepsilon}-\nabla u-\left(\nabla w_{\varepsilon}-\nabla w\right) T_{k}(u)\right|^{2}\right)^{\frac{p}{2}} \\
& \quad+c\left(\int_{\{|u|>k\} \cap \omega}|u|^{\frac{2 p}{2-p}} d x\right)^{1-\frac{p}{2}} .
\end{aligned}
$$

Since $u \in L^{\frac{2 p}{2-p}}(\omega)$ by the Sobolev embedding, passing successively to the limits $\varepsilon \rightarrow 0$ and $k \rightarrow \infty$ in (2.52) owing to convergence (2.51) we obtain the strong convergence (2.30).

Let $r \in[1, p)$. Since $w_{\varepsilon}-w$ strongly converges to 0 in $L^{\frac{2 r}{2-r}}(\omega)$, by the Hölder inequality the sequence $\left(w_{\varepsilon}-w\right) \nabla u$ strongly converges to 0 in $L^{r}(\omega)^{N}$. Finally, this combined with (2.30) implies the corrector result (2.31). 


\subsection{A counter-example}

In this section $\Omega$ is a regular bounded open set of $\mathbb{R}^{2}$, and $Y:=\left(-\frac{1}{2}, \frac{1}{2}\right)^{2}$. For fixed $R \in\left(0, \frac{1}{2}\right)$ and $\mu>0$, let $r_{\varepsilon} \in(0, R)$ be defined by the equality

$$
\frac{2 \pi}{\varepsilon^{2}\left|\ln r_{\varepsilon}\right|}=\mu \text {. }
$$

Let $W_{\varepsilon}$ be the $Y$-periodic function and $w_{\varepsilon}$ be the $\varepsilon Y$-periodic function defined by

$$
W_{\varepsilon}(y):=\left\{\begin{array}{ll}
\frac{\ln r-\ln r_{\varepsilon}}{\ln R-\ln r_{\varepsilon}} & \text { if } r:=|y| \in\left(r_{\varepsilon}, R\right) \\
0 & \text { si } r \leq r_{\varepsilon} \\
1 & \text { si } r \geq R,
\end{array} \quad y \in Y, w_{\varepsilon}(x):=W_{\varepsilon}\left(\frac{x}{\varepsilon}\right), x \in \mathbb{R}^{2} .\right.
$$

Note that by (2.53) we have

$$
\frac{1}{\varepsilon^{2}} \int_{Y}\left|\nabla W_{\varepsilon}\right|^{2} d y=\frac{2 \pi}{\varepsilon^{2} \ln \left(R / r_{\varepsilon}\right)} \underset{\varepsilon \rightarrow 0}{\longrightarrow} \mu .
$$

We then consider the drift $b_{\varepsilon}$ defined by

$$
b_{\varepsilon}(x):=\nabla w_{\varepsilon}(x)=\frac{1}{\varepsilon} \nabla W_{\varepsilon}\left(\frac{x}{\varepsilon}\right), \quad \text { for } x \in \mathbb{R}^{2} .
$$

Taking into account (2.53) it is easy to check that

$$
w_{\varepsilon} \longrightarrow 1 \text { weakly in } H^{1}(\Omega) \text { and weakly-* in } L^{\infty}(\Omega) .
$$

Let $f$ be a non-zero function in $L^{2}(\Omega)$. We study the asymptotic behavior of the equation (2.4) with the drift $b_{\varepsilon}$ of (2.56), i.e.

$$
-\Delta u_{\varepsilon}+\nabla w_{\varepsilon} \cdot \nabla u_{\varepsilon}+\operatorname{div}\left(\nabla w_{\varepsilon} u_{\varepsilon}\right)=f \quad \text { in } \mathcal{D}^{\prime}(\Omega) .
$$

We have the following result:

Theorem 2.6. The solution $u_{\varepsilon}$ of (2.58) weakly converges in $H_{0}^{1}(\Omega)$ to the solution $u$ of the equation

$$
-\Delta u+\gamma u=f \quad \text { in } \mathcal{D}^{\prime}(\Omega), \quad \text { where } \quad \gamma:=\frac{3\left(e^{2}-1\right)}{4\left(e^{2}+1\right)} \mu<\mu .
$$

Remark 2.7. Using the periodicity we can check that the sequence $\left|b_{\varepsilon}\right|^{2}=\left|\nabla w_{\varepsilon}\right|^{2}$ converges in the weak-* sense of measures on $\Omega$ - but not weakly in $L^{1}(\Omega)$ - to the constant $\mu$ defined by (2.53). Theorem 2.6 can thus be regarded as a counterexample to the statement of Theorem 2.4 without the equi-integrability assumption on the drift $b_{\varepsilon}$ in $L^{2}(\Omega)^{2}$. Indeed, the conclusion of Theorem 2.4 would give a limit equation (2.59), with $\gamma=\mu$. 
Proof of Theorem 2.6. The proof is divided into two steps. In the first step we construct an oscillating test function $z_{\varepsilon}$ which solves equation (2.64) below. In the second step we determine the limit equation (2.59).

First step. Construction of an oscillating test function.

Denote by $Q_{r}$ the disk of radius $r$ centered at the origin. Consider the unique solution $Z_{\varepsilon}$ in $H^{1}\left(Q_{R}\right)$ of the equation

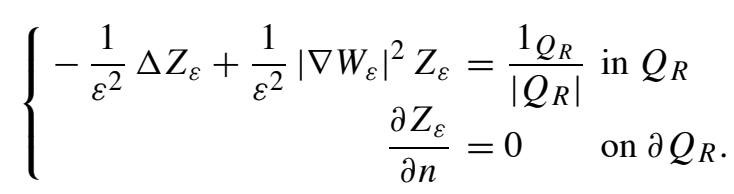

The function $Z_{\varepsilon}$ is radial and can be computed explicitly. Using the Laplace operator in polar coordinates and $\left|\nabla W_{\varepsilon}\right|^{2}=\alpha_{\varepsilon}^{2} r^{-2} 1_{Q_{R} \backslash \bar{Q}_{r_{\varepsilon}}}$, we get

$$
\begin{aligned}
Z_{\varepsilon}(r)= & \begin{cases}-\frac{\varepsilon^{2}}{4 \pi R^{2}} r^{2}+c_{\varepsilon} & \text { if } r \in\left(0, r_{\varepsilon}\right] \\
a_{\varepsilon} r^{\alpha_{\varepsilon}}+b_{\varepsilon} r^{-\alpha_{\varepsilon}}+\frac{\varepsilon^{2}}{\pi R^{2}\left(\alpha_{\varepsilon}^{2}-3\right)} r^{2} & \text { if } r \in\left(r_{\varepsilon}, R\right],\end{cases} \\
& \text { where } \alpha_{\varepsilon}:=\frac{1}{\ln \left(R / r_{\varepsilon}\right)} .
\end{aligned}
$$

The constants $a_{\varepsilon}, b_{\varepsilon}, c_{\varepsilon}$ are determined owing to the boundary condition on $\partial Q_{R}$ and to the transmission conditions on $\partial Q_{r_{\varepsilon}}$, i.e.

$$
Z_{\varepsilon}^{\prime}(R)=0 \quad \text { and } \quad Z_{\varepsilon}\left(r_{\varepsilon}^{+}\right)=Z_{\varepsilon}\left(r_{\varepsilon}^{-}\right), \quad Z_{\varepsilon}^{\prime}\left(r_{\varepsilon}^{+}\right)=Z_{\varepsilon}^{\prime}\left(r_{\varepsilon}^{-}\right) .
$$

We extend $Z_{\varepsilon}$ by the constant value $Z_{\varepsilon}(R)$ in $Y \backslash \bar{Q}_{R}$, and by $Y$-periodicity in the whole space $\mathbb{R}^{2}$. The $Y$-periodic extension is still denoted by $Z_{\varepsilon}$. An explicit computation combined with (2.53) yields

$$
Z_{\varepsilon} \longrightarrow \bar{Z}:=\frac{4\left(e^{2}+1\right)}{3\left(e^{2}-1\right)} \frac{1}{\mu} \quad \text { strongly in } H_{\sharp}^{1}(Y) .
$$

As a consequence of (2.60), (2.61) the rescaled function $z_{\varepsilon}(x):=Z_{\varepsilon}\left(\frac{x}{\varepsilon}\right)$ is solution of the equation

$$
-\Delta z_{\varepsilon}+\left|\nabla w_{\varepsilon}\right|^{2} z_{\varepsilon}=\chi_{Q_{R}}^{\sharp}\left(\frac{x}{\varepsilon}\right) \quad \text { in } \mathcal{D}^{\prime}\left(\mathbb{R}^{2}\right),
$$

where $\chi_{Q_{R}}^{\sharp}$ is the $Y$-periodic function agreeing with $\frac{1 Q_{R}}{\left|Q_{R}\right|}$ in the period cell $Y$. Moreover, the following convergences hold

$$
z_{\varepsilon} \longrightarrow \bar{Z} \text { weakly in } H^{1}(\Omega) \text { and } \chi_{Q_{R}}^{\sharp}\left(\frac{x}{\varepsilon}\right) \longrightarrow 1 \text { weakly-* in } L^{\infty}(\Omega),
$$

where the constant $\bar{Z}$ is defined by (2.63). 
Second step. Determination of the limit equation (2.59).

Define the function $v_{\varepsilon}:=e^{1-w_{\varepsilon}} u_{\varepsilon}$. Then, equation (2.58) is equivalent to

$$
-\Delta v_{\varepsilon}+\left|\nabla w_{\varepsilon}\right|^{2} v_{\varepsilon}=e^{1-w_{\varepsilon}} f \quad \text { in } \mathcal{D}^{\prime}(\Omega) .
$$

G. Dal Maso, A. Garroni [7] proved that this class of equations is stable under homogenization. In the present case, the use of the oscillating test function $z_{\varepsilon}$ will allow us to obtain the limit equation (2.59).

On the one hand, choosing $v=w_{\varepsilon}$ in (2.8) we get

$$
\int_{\Omega}\left|\nabla w_{\varepsilon}\right|^{2} u_{\varepsilon}^{2} d x-\int_{\Omega} \nabla w_{\varepsilon} \nabla u_{\varepsilon} u_{\varepsilon} d x=\int_{\Omega}\left|\nabla u_{\varepsilon}\right|^{2} w_{\varepsilon} d x-\int_{\Omega} f w_{\varepsilon} u_{\varepsilon} d x \leq c,
$$

since $u_{\varepsilon}$ is bounded in $H_{0}^{1}(\Omega)$ and $0 \leq w_{\varepsilon} \leq 1$. Then, by the Cauchy-Schwarz inequality we have

$$
\begin{aligned}
\int_{\Omega}\left|\nabla w_{\varepsilon}\right|^{2} u_{\varepsilon}^{2} d x & \leq c+c\left(\int_{\Omega}\left|\nabla u_{\varepsilon}\right|^{2} d x\right)^{\frac{1}{2}}\left(\int_{\Omega}\left|\nabla w_{\varepsilon}\right|^{2} u_{\varepsilon}^{2} d x\right)^{\frac{1}{2}} \\
& \leq c+c^{\prime}\left(\int_{\Omega}\left|\nabla w_{\varepsilon}\right|^{2} u_{\varepsilon}^{2} d x\right)^{\frac{1}{2}},
\end{aligned}
$$

hence $u_{\varepsilon} \nabla w_{\varepsilon}$ is bounded in $L^{2}(\Omega)^{2}$. This combined with convergence (2.57) implies that $v_{\varepsilon}$ weakly converges to $u$ in $H_{0}^{1}(\Omega)$.

On the other hand, for $\varphi \in C_{c}^{\infty}(\Omega)$, putting the functions $\varphi z_{\varepsilon}$ in (2.66) and $\varphi v_{\varepsilon}$ in (2.64), taking the difference of the two equalities, and passing to the limit owing to convergences (2.65) we obtain the equality

$$
\int_{\Omega} \nabla u \cdot \nabla \varphi \bar{Z} d x+\int_{\Omega} \varphi u d x=\int_{\Omega} f \varphi \bar{Z} d x, \quad \text { for any } \varphi \in C_{c}^{\infty}(\Omega) .
$$

which is the variational formulation of equation (2.59), with $\gamma=\bar{Z}^{-1}$.

\section{A Stokes equation with a drift term}

\subsection{The classical case}

In $[14,15] \mathrm{L}$. Tartar noted that the nonlinear term of the three-dimensional NavierStokes equation for the divergence free velocity $u$ reads as

$$
(u \cdot \nabla) u=\operatorname{Div}(u \otimes u)=\operatorname{curl}(u) \times u+\nabla\left(\frac{1}{2}|u|^{2}\right) .
$$

This led him to study the perturbed Stokes equation

$$
-\Delta u+\operatorname{curl}(v) \times u+\nabla p=f,
$$


where a given vector-valued function $v$ replaced the velocity $u$ of the Navier-Stokes equation. The equivalent of transformation (3.1) in two-dimension is

$$
\begin{gathered}
\operatorname{Div}(u \otimes u)=\operatorname{curl}(u) J u+\nabla\left(\frac{1}{2}|u|^{2}\right), \\
\text { where } \operatorname{curl}(u):=\partial_{1} u_{2}-\partial_{2} u_{1} \quad \text { and } \quad J:=\left(\begin{array}{cc}
0 & -1 \\
1 & 0
\end{array}\right) .
\end{gathered}
$$

More generally equality (3.3) extends for any divergence free functions $u, v$ to the following one

$$
\operatorname{curl}(v) J u=\operatorname{Div}(v \otimes u)+(D u)^{T} v-\nabla(v \cdot u) .
$$

Similarly to (3.2) this leads us to the two-dimensional perturbed Stokes equation

$$
-\Delta u+\operatorname{curl}(v) J u+\nabla p=f .
$$

Let $\Omega$ be a bounded domain of $\mathbb{R}^{2}$. Let $v_{\varepsilon}$ be a sequence in $L^{\infty}(\Omega)^{2}$ and let $f$ be a distribution in $H^{-1}(\Omega)^{2}$. Consider the perturbed Stokes equation

$$
\begin{cases}-\Delta u_{\varepsilon}+\operatorname{curl}\left(v_{\varepsilon}\right) J u_{\varepsilon}+\nabla p_{\varepsilon}=f & \text { in } \Omega \\ \operatorname{div}\left(u_{\varepsilon}\right)=0 & \text { in } \Omega \\ u_{\varepsilon}=0 & \text { on } \partial \Omega .\end{cases}
$$

In the three-dimensional case where $\operatorname{curl}\left(v_{\varepsilon}\right) \times u_{\varepsilon}$ replaces $\operatorname{curl}\left(v_{\varepsilon}\right) J u_{\varepsilon}, \mathrm{L}$. Tartar [15] derived a Stokes equation with a Brinkman law under the assumption that $v_{\varepsilon}$ is bounded in $L^{3}(\Omega)^{3}$ (see Introduction). Mimicking the Tartar approach in dimension two we can derive a similar homogenized equation using the test function $w_{\varepsilon}^{\lambda}$, for $\lambda \in \mathbb{R}^{2}$, solution of the Stokes equation

$$
\begin{cases}-\Delta w_{\varepsilon}^{\lambda}+\operatorname{Div}\left(\left(v_{\varepsilon}-v\right) \otimes \lambda\right)+\nabla q_{\varepsilon}^{\lambda}=0 & \text { in } \Omega \\ \operatorname{div}\left(w_{\varepsilon}^{\lambda}\right)=0 & \text { in } \Omega \\ w_{\varepsilon}^{\lambda}=0 & \text { on } \partial \Omega .\end{cases}
$$

Then, we have the following result:

Theorem 3.1. Assume that $v_{\varepsilon}$ is bounded in $L^{r}(\Omega)^{2}$, with $r>2$. Then, the solution $u_{\varepsilon}$ of (3.6) weakly converges in $H_{0}^{1}(\Omega)$ to the solution $u$ of the Brinkman equation

$$
\begin{cases}-\Delta u+\operatorname{curl}(v) J u+\nabla p+M u=f & \text { in } \Omega \\ \operatorname{div}(u)=0 & \text { in } \Omega \\ u=0 & \text { on } \partial \Omega,\end{cases}
$$

where $M$ is the positive definite symmetric matrix-valued function defined by $\left\{\begin{array}{ll}\left(D w_{\varepsilon}^{\lambda}\right)^{T} v_{\varepsilon} \longrightarrow M \lambda & \text { weakly in } L^{\frac{2 r}{2+r}}(\Omega)^{2} \text { and in } L_{\text {loc }}^{\frac{r}{2}}(\Omega)^{2} \\ D w_{\varepsilon}^{\lambda} \cdot D w_{\varepsilon}^{\mu} \longrightarrow M \lambda \cdot \mu & \text { weakly-* in } \mathcal{M}(\Omega)^{2} \text { and in } L_{\text {loc }}^{\frac{r}{2}}(\Omega)^{2},\end{array} \quad\right.$ for $\lambda, \mu \in \mathbb{R}^{2}$. 
Moreover, the zero-order term of (3.8) is given by the convergences

$$
\begin{cases}\left(D u_{\varepsilon}\right)^{T}\left(v_{\varepsilon}-v\right) \longrightarrow M u & \text { weakly in } L^{\frac{2 r}{2+r}}(\Omega)^{2} \\ D u_{\varepsilon}: D w_{\varepsilon}^{\lambda} \longrightarrow M u \cdot \lambda & \text { weakly-* in } \mathcal{M}(\Omega) \text { and in } L_{\mathrm{loc}}^{\frac{2 r}{2+r}}(\Omega)^{2}\end{cases}
$$

Proof. By the representation formula (3.4) we have

$$
\operatorname{curl}\left(v_{\varepsilon}\right) J u_{\varepsilon}=\left(D u_{\varepsilon}\right)^{T} v_{\varepsilon}+\operatorname{Div}\left(v_{\varepsilon} \otimes u_{\varepsilon}\right)-\nabla\left(v_{\varepsilon} \cdot u_{\varepsilon}\right) .
$$

Hence, the variational formulation of (3.6) reads as

$$
\begin{array}{r}
\int_{\Omega} D u_{\varepsilon}: D \varphi d x+\int_{\Omega}\left(D u_{\varepsilon}\right)^{T} v_{\varepsilon} \cdot \varphi d x-\int_{\Omega}\left(v_{\varepsilon} \otimes u_{\varepsilon}\right): D \varphi d x=\left\langle f, u_{\varepsilon}\right\rangle_{H^{-1}(\Omega)^{2}, H_{0}^{1}(\Omega)^{2}} \\
\text { for any } \varphi \in H_{0}^{1}(\Omega)^{2}, \operatorname{div}(\varphi)=0 .
\end{array}
$$

By the Lax-Milgram theorem there exists a unique divergence free function $u_{\varepsilon} \in$ $H_{0}^{1}(\Omega)^{2}$ solution of (3.12). Then, putting the velocity $u_{\varepsilon}$ as test function in (3.12) it follows that

$$
\int_{\Omega}\left|D u_{\varepsilon}\right|^{2} d x=\left\langle f, u_{\varepsilon}\right\rangle_{H^{-1}(\Omega)^{2}, H_{0}^{1}(\Omega)^{2}},
$$

which implies that $u_{\varepsilon}$ is bounded in $H_{0}^{1}(\Omega)^{2}$. Let $\omega$ be a regular domain of $\Omega$. Applying (3.12) to divergence free functions in $H_{0}^{1}(\omega)^{2}$, there exists a unique $p_{\varepsilon}$ in $L^{2}(\omega) / \mathbb{R}$ such that equation (3.6) holds in $\mathcal{D}^{\prime}(\omega)^{2}$. Moreover, by (3.11) and the boundedness of $v_{\varepsilon}$ in $L^{r}(\Omega)^{2}$ the sequence $\nabla p_{\varepsilon}$ is bounded in $H^{-1}(\omega)^{2}$. Hence, due to the regularity of $\omega$ the sequence $p_{\varepsilon}$ is bounded in $L^{2}(\omega)$. Then, considering an exhaustive sequence of regular domains the union of which is $\Omega$, we can construct in $\Omega$ a pressure $p_{\varepsilon}$ which is bounded in $L_{\text {loc }}^{2}(\Omega)$. Therefore, up to a subsequence the following convergences hold

$$
\begin{cases}u_{\varepsilon} \longrightarrow u & \text { weakly in } H_{0}^{1}(\Omega)^{2} \\ p_{\varepsilon} \longrightarrow p & \text { weakly in } L_{\mathrm{loc}}^{2}(\Omega) / \mathbb{R}\end{cases}
$$

Now, in view of (3.11) it is enough to determine the limit of the term $\left(D u_{\varepsilon}\right)^{T} v_{\varepsilon}$. By the regularity results for the Stokes equation (see, e.g., [10] Theorem 2, p. 67) the sequences $w_{\varepsilon}^{\lambda}$ and $q_{\varepsilon}^{\lambda}$ satisfy

$$
\left\{\begin{array}{l}
w_{\varepsilon}^{\lambda} \longrightarrow 0 \text { weakly in } H^{1}(\Omega)^{2} \text { and in } W_{\text {loc }}^{1, r}(\Omega)^{2} \\
q_{\varepsilon}^{\lambda} \longrightarrow 0 \text { weakly in } L^{2}(\Omega) / \mathbb{R} \text { and in } L_{\text {loc }}^{r}(\Omega) / \mathbb{R}
\end{array}\right.
$$

which imply convergence (3.9). Let $\varphi \in C_{c}^{\infty}(\Omega)$. Following the Tartar method we put $\varphi w_{\varepsilon}^{\lambda}$ in equation (3.6) and $\varphi u_{\varepsilon}$ in equation (3.7). Then, from the representation 
(3.11), the convergences (3.14), (3.15) and the boundedness of $v_{\varepsilon}$ in $L^{r}(\Omega)$ we deduce that

$\begin{cases}\int_{\Omega} D u_{\varepsilon}: D w_{\varepsilon}^{\lambda} \varphi d x-\int_{\Omega}\left(v_{\varepsilon} \otimes u_{\varepsilon}\right): D w_{\varepsilon}^{\lambda} \varphi d x=o(1) & \\ \int_{\Omega} D w_{\varepsilon}^{\lambda}: D u_{\varepsilon} \varphi d x-\int_{\Omega}\left(\left(v_{\varepsilon}-v\right) \otimes \lambda\right): D u_{\varepsilon} \varphi d x=o(1), & \text { for any } \varphi \in C_{c}^{\infty}(\Omega),\end{cases}$

hence

$$
\left\{\begin{array}{l}
D u_{\varepsilon}: D w_{\varepsilon}^{\lambda}-\left(D w_{\varepsilon}^{\lambda}\right)^{T} v_{\varepsilon} \cdot u_{\varepsilon} \longrightarrow 0 \\
\left(D u_{\varepsilon}\right)^{T}\left(v_{\varepsilon}-v\right) \cdot \lambda-\left(D w_{\varepsilon}^{\lambda}\right)^{T} v_{\varepsilon} \cdot u_{\varepsilon} \longrightarrow 0
\end{array} \quad \text { in } \mathcal{D}^{\prime}(\Omega) .\right.
$$

By virtue of the strong convergence of $u_{\varepsilon}$ in any $L^{s}(\Omega)^{2}$ space for $s \in(1, \infty)$, convergences (3.16) and (3.9) imply (3.10). This combined with (3.11) yields finally the limit problem (3.8).

Remark 3.2. It can be shown that

$$
M(x)=\int_{S^{1}}[\operatorname{tr}(\boldsymbol{\mu}(x, d \xi))-\boldsymbol{\mu}(x, d \xi) \xi \cdot \xi] \xi \otimes \xi
$$

where $\boldsymbol{\mu}$ is the matrix-valued $H$-measure of the sequence $v_{\varepsilon}$ (see $[16,17]$ ).

The case where $v_{\varepsilon}$ is only bounded in $L^{2}(\Omega)^{2}$ is much more delicate. On the one hand, under additional assumptions we will extend the Tartar result when $v_{\varepsilon}$ is bounded and equi-integrable in $L^{2}(\Omega)^{2}$. On the other hand, we will give an example of a sequence $v_{\varepsilon}$ for which the homogenized Brinkman equation is not the one obtained by the Tartar procedure.

\subsection{The case under an equi-integrability condition}

In this section we make the following weaker assumption on the drift,

$$
v_{\varepsilon} \longrightarrow v \text { weakly in } L^{2}(\Omega)^{2} \text { and } v_{\varepsilon} \text { is equi-integrable in } L^{2}(\Omega)^{2} .
$$

Then, we have the following extension of Theorem 3.1:

\section{Theorem 3.3.}

i) Under the equi-integrability assumption (3.18) the solution $u_{\varepsilon}$ of (2.4) weakly converges in $H_{0}^{1}(\Omega)$ to the solution $u$ of equation (3.8) with

$$
\int_{\Omega} M u \cdot u d x \leq\langle f, u\rangle_{H^{-1}(\Omega)^{2}, H_{0}^{1}(\Omega)^{2}}-\int_{\Omega}|D u|^{2} d x,
$$

where $M$ is the positive definite symmetric matrix-valued function defined by

$$
\left\{\begin{array}{ll}
\left(D w_{\varepsilon}^{\lambda}\right)^{T} v_{\varepsilon} \longrightarrow M \lambda & \text { weakly in } L^{1}(\Omega)^{2} \\
D w_{\varepsilon}^{\lambda}: D w_{\varepsilon}^{\mu} \longrightarrow M \lambda \cdot \mu & \text { weakly-* in } \mathcal{M}(\Omega)^{2},
\end{array} \quad \text { for } \lambda, \mu \in \mathbb{R}^{2} .\right.
$$


ii) Also assume that $\Omega$ has a Lipschitz boundary, $v \in L^{r}(\Omega)^{2}$, with $r>2$, and $M \in L^{m}(\Omega)^{2 \times 2}$, with $m>1$. Then, we have the equality

$$
\int_{\Omega}|D u|^{2} d x+\int_{\Omega} M u \cdot u d x=\langle f, u\rangle_{H^{-1}(\Omega)^{2}, H_{0}^{1}(\Omega)^{2}},
$$

and there exists a unique solution $u \in H_{0}^{1}(\Omega)^{2}$ of equation (3.8), with $M u \cdot u \in$ $L^{1}(\Omega)$.

Moreover, we have the corrector result

$$
u_{\varepsilon}-u-W_{\varepsilon} u \longrightarrow 0 \text { strongly in } W^{1,1}(\Omega)^{2},
$$

where $W_{\varepsilon}$ is the matrix-valued function defined by

$$
W_{\varepsilon} \lambda:=w_{\varepsilon}^{\lambda}, \quad \text { for } \lambda \in \mathbb{R}^{2} .
$$

Remark 3.4. Contrary to Theorem 2.4, in the part ii) of Theorem 3.3 we need to assume a higher integrability for the matrix-valued $M$. Indeed, we cannot apply a truncation principle on $M u \cdot u$. Moreover, the regularity of $\Omega$ is necessary to obtain the density of the smooth divergence free functions in the space of the divergence free functions of $H_{0}^{1}(\Omega)^{2}$.

Proof of Theorem 3.3. As in the proof of Theorem 3.3 the sequence $u_{\varepsilon}$ is bounded in $H_{0}^{1}(\Omega)^{2}$, and thus in any $L^{s}(\Omega)^{2}$ space. Then, in view of (3.11) and (3.6) together with the boundedness of $u_{\varepsilon}$ and $v_{\varepsilon}$ the sequence $\nabla p_{\varepsilon}$ is bounded in $L^{1}(\Omega)^{2}+$ $W^{-1, r}(\Omega)^{2}$ for any $r \in(1,2)$. Hence, thanks to the embedding of $L_{\text {loc }}^{1}(\Omega)$ into $W_{\text {loc }}^{-\sigma, r}(\Omega)$ for any $r>1$ and $\sigma>2 / r^{\prime}$, the sequence $p_{\varepsilon}$ is bounded in $L_{\text {loc }}^{r}(\Omega) / \mathbb{R}$ for any $r \in(1,2)$. Therefore, up to a subsequence we have the convergences

$$
\left\{\begin{array}{l}
u_{\varepsilon} \longrightarrow u \text { weakly in } H_{0}^{1}(\Omega)^{2} \\
p_{\varepsilon} \longrightarrow p \text { weakly in } L_{\text {loc }}^{r}(\Omega) / \mathbb{R} \text {, for any } r \in(1,2) .
\end{array}\right.
$$

The problem is to determine the vector-valued distribution $v$ defined by

$$
\operatorname{curl}\left(v_{\varepsilon}\right) J u_{\varepsilon}-\operatorname{curl}(v) J u \longrightarrow v \quad \text { in } \mathcal{D}^{\prime}(\Omega)^{2} .
$$

Taking into account the representation formula (3.11) and the equi-integrability of $v_{\varepsilon}$ in $L^{2}(\Omega)^{2}, v$ is actually in $L^{1}(\Omega)^{2}$, and is given by

$$
\left(D u_{\varepsilon}\right)^{T}\left(v_{\varepsilon}-v\right) \longrightarrow v \quad \text { weakly in } L^{1}(\Omega)^{2},
$$

so that $u$ is solution of the equation

$$
-\Delta u+v+\operatorname{curl}(v) J u+\nabla p=f \quad \text { in } \mathcal{D}^{\prime}(\Omega) .
$$

From now on the proof follows the same scheme as the one of Theorem 2.4 using a representation of the velocity and the pressure owing to the parametrix $P$ of (2.35). 
The proof is divided into five steps. The first step deals with a double parametrix method for both $u_{\varepsilon}$ and $p_{\varepsilon}$, which allows us to derive a strong approximation of $D u_{\varepsilon}$. In the second step we compute the limit $\sigma^{k}$ of the sequence $\left(D u_{\varepsilon}\right)^{T} v_{\varepsilon}^{k}$, where $v_{\varepsilon}^{k}$ is a truncation of $v_{\varepsilon}$ for a fixed $k>0$. In the third step we obtain the limit equation (3.8). In the fourth step we prove inequality (3.19). The fifth step is devoted to the proof of equality (3.21) and the corrector result (3.22).

First step. The double parametrix method.

Consider the parametrix $P(2.35)$ for the Laplace operator. Abusively we denote by $\Delta$ the vector-valued Laplace operator as well as by $P$ the associated vector-valued parametrix each component of which is defined by (2.35). Taking the divergence of equation (3.6) we have

$$
\Delta p_{\varepsilon}=\operatorname{div}(f)-\operatorname{div}\left(\operatorname{curl}\left(v_{\varepsilon}\right) J u_{\varepsilon}\right) \text { in } \Omega,
$$

hence by (2.36)

$$
p_{\varepsilon}=P\left(\operatorname{div}(f)-\operatorname{div}\left[\operatorname{curl}\left(v_{\varepsilon}\right) J u_{\varepsilon}\right]\right)+K\left(p_{\varepsilon}\right) \quad \text { in } \Omega .
$$

Substituting $p_{\varepsilon}$ by the right-hand side of (3.28) in (3.6) it follows that $\Delta u_{\varepsilon}=\operatorname{curl}\left(v_{\varepsilon}\right) J u_{\varepsilon}-\nabla P\left(\operatorname{div}\left[\operatorname{curl}\left(v_{\varepsilon}\right) J u_{\varepsilon}\right]\right)+\nabla P(\operatorname{div}(f))-f+\nabla K\left(p_{\varepsilon}\right) \quad$ in $\Omega$, hence again by (2.36) we have in $\Omega$

$$
\begin{aligned}
u_{\varepsilon}= & P\left(\operatorname{curl}\left(v_{\varepsilon}\right) J u_{\varepsilon}-\nabla P\left(\operatorname{div}\left[\operatorname{curl}\left(v_{\varepsilon}\right) J u_{\varepsilon}\right]\right)\right) \\
& +P(\nabla P(\operatorname{div}(f))-f)+L\left(u_{\varepsilon}, p_{\varepsilon}\right),
\end{aligned}
$$

where $L$ is a $C^{\infty}$-kernel operator acting on the pair $\left(u_{\varepsilon}, p_{\varepsilon}\right)$. Using the representation (3.11) of curl $\left(v_{\varepsilon}\right) J u_{\varepsilon}$, and setting

$$
g_{\varepsilon}:=\operatorname{Div}\left(\left(v_{\varepsilon}-v\right) \otimes u_{\varepsilon}\right)-\nabla\left(\left(v_{\varepsilon}-v\right) \cdot u_{\varepsilon}\right),
$$

we get

$$
u_{\varepsilon}=P\left(\left(D u_{\varepsilon}\right)^{T} v_{\varepsilon}+g_{\varepsilon}-\nabla P\left(\operatorname{div}\left[\left(D u_{\varepsilon}\right)^{T} v_{\varepsilon}+g_{\varepsilon}\right]\right)\right)+F\left(u_{\varepsilon}, p_{\varepsilon}\right),
$$

where

$$
\begin{aligned}
F(\zeta, \theta):= & P(\operatorname{Div}(v \otimes \zeta)-\nabla(v \cdot \zeta)-f-\nabla P(\operatorname{div}[\operatorname{Div}(v \otimes \zeta)-\nabla(v \cdot \zeta)-f])) \\
& +L(\zeta, \theta)
\end{aligned}
$$

Note that by (2.37) we have

$$
F\left(u_{\varepsilon}, p_{\varepsilon}\right) \longrightarrow F(u, p) \quad \text { strongly in } W_{\text {loc }}^{1, r}(\Omega), \quad \text { for any } r \in(1,2) .
$$


Moreover, by (3.26) the sequence $\left(D u_{\varepsilon}\right)^{T} v_{\varepsilon}$ weakly converges to $v+(D u)^{T} v$ in $L^{1}(\Omega)^{2}$ which is compactly embedded in $W_{\text {loc }}^{-1, r}(\Omega)^{2}$ for any $r \in(1,2)$. Hence, as in the first step of the proof of Theorem 2.4, from (3.31) and the two previous convergences we deduce, for any $r \in(1,2)$, the strong convergence

$$
\begin{gathered}
u_{\varepsilon}-P\left(g_{\varepsilon}-\nabla P\left(\operatorname{div}\left(g_{\varepsilon}\right)\right)\right) \longrightarrow P\left(v+(D u)^{T} v-\nabla P\left(\operatorname{div}\left[v+(D u)^{T} v\right]\right)\right) \\
+F(u, p) \quad \text { strongly in } W_{\text {loc }}^{1, r}(\Omega)^{2}
\end{gathered}
$$

Second step. Determination of the limit $\sigma^{k}$ of $\left(D u_{\varepsilon}\right)^{T} v_{\varepsilon}^{k}$.

Fix $r \in(1,2)$ such that (3.33) holds. Set

$$
z_{\varepsilon}:=P\left(g_{\varepsilon}-\nabla P\left(\operatorname{div}\left(g_{\varepsilon}\right)\right)\right) \quad \text { and } \quad q_{\varepsilon}:=P\left(\operatorname{div}\left(g_{\varepsilon}\right)\right) .
$$

In view of (3.30) the sequence $g_{\varepsilon}$ weakly converges to 0 in $W^{-1, r}(\Omega)^{2}$, hence by (2.37) we have

$$
z_{\varepsilon} \longrightarrow 0 \text { weakly in } W_{\text {loc }}^{1, r}(\Omega)^{2} \text { and } q_{\varepsilon} \longrightarrow 0 \text { weakly in } L_{\mathrm{loc}}^{r}(\Omega) / \mathbb{R} \text {. }
$$

Moreover, by (2.36) we have

$$
\Delta z_{\varepsilon}=g_{\varepsilon}-\nabla q_{\varepsilon}-K^{\prime}\left(g_{\varepsilon}\right) \quad \text { and } \quad \Delta q_{\varepsilon}=\operatorname{div}\left(g_{\varepsilon}\right)-K^{\prime}\left(q_{\varepsilon}\right) \quad \text { in } \Omega,
$$

hence

$$
\Delta\left(\operatorname{div}\left(z_{\varepsilon}\right)\right)=K^{\prime}\left(q_{\varepsilon}\right)-\operatorname{div}\left(K^{\prime}\left(g_{\varepsilon}\right)\right) \longrightarrow 0 \quad \text { strongly in } L_{\text {loc }}^{r}(\Omega)^{2} \text {, say. }
$$

This combined with the first convergence of (3.35) and (2.37) yields

$$
\operatorname{div}\left(z_{\varepsilon}\right) \longrightarrow 0 \quad \text { strongly in } W_{\text {loc }}^{2, r}(\Omega)^{2} .
$$

On the other hand, set $v_{\varepsilon}^{k}:=v_{\varepsilon} 1_{\left\{\left|v_{\varepsilon}\right|<k\right\}}$, for a positive integer $k$. Up to a subsequence of $\varepsilon$ still denoted by $\varepsilon, v_{\varepsilon}^{k}$ weakly converges to some function $v^{k}$ in $L^{2}(\Omega)^{2}$ for any $k$. Consider for $\lambda \in \mathbb{R}^{2}$, the solutions $w_{\varepsilon}^{\lambda, k}$ and $q_{\varepsilon}^{\lambda, k}$ of the Stokes problem

$$
\begin{cases}-\Delta w_{\varepsilon}^{\lambda, k}+\operatorname{Div}\left(\left(v_{\varepsilon}^{k}-v^{k}\right) \otimes \lambda\right)+\nabla q_{\varepsilon}^{\lambda, k}=0 & \text { in } \Omega \\ \operatorname{div}\left(w_{\varepsilon}^{\lambda, k}\right)=0 & \text { in } \Omega \\ w_{\varepsilon}^{\lambda, k}=0 & \text { on } \partial \Omega\end{cases}
$$

which consists in an approximation of equation (3.7). By the regularity results for the Stokes equation (see, e.g., [10]) we have

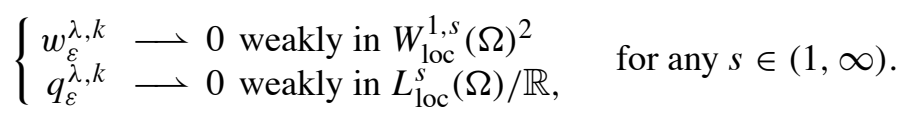


Take $s:=r^{\prime}$ and choose good oscillating test functions as Tartar did (see [13, Appendix]). Let $\varphi \in C_{c}^{\infty}(\Omega)$. Putting $\varphi w_{\varepsilon}^{\lambda, k}$ in the first equation of (3.36) and $\varphi z_{\varepsilon}$ in equation (3.38), and using the definition (3.30) of $g_{\varepsilon}$ and the convergences (3.35), (3.37), (3.39) we have

$$
\left(D z_{\varepsilon}\right)^{T}\left(v_{\varepsilon}^{k}-v^{k}\right) \cdot \lambda-\left(D w_{\varepsilon}^{\lambda, k}\right)^{T}\left(v_{\varepsilon}-v\right) \cdot u_{\varepsilon} \longrightarrow 0 \quad \text { weakly in } \mathcal{D}^{\prime}(\Omega)^{2} .
$$

Hence, since $D z_{\varepsilon}$ weakly converges to 0 in $L^{r}(\Omega)^{2 \times 2}$, we deduce that

$$
\left(D z_{\varepsilon}\right)^{T} v_{\varepsilon}^{k} \longrightarrow M^{k} u \quad \text { weakly in } \mathcal{D}^{\prime}(\Omega)^{2},
$$

where the matrix-valued function $M^{k}$ is defined by

$$
\left(D w_{\varepsilon}^{\lambda, k}\right)^{T} v_{\varepsilon} \longrightarrow M^{k} \lambda \quad \text { weakly in } L_{\text {loc }}^{s}(\Omega)^{2}, \quad \text { for any } s \in[1,2) .
$$

Now, we are able to determine the limit $\sigma^{k}$ of the sequence $\left(D u_{\varepsilon}\right)^{T} v_{\varepsilon}^{k}$ in $L^{2}(\Omega)^{2}$. With the definition (3.34) of $z_{\varepsilon}$ the strong convergence (3.33) implies that

$$
\begin{aligned}
& \left(D u_{\varepsilon}\right)^{T} v_{\varepsilon}^{k}-\left(D z_{\varepsilon}\right)^{T} v_{\varepsilon}^{k} \longrightarrow\left[D P\left(v+(D u)^{T} v-\nabla P\left(\operatorname{div}\left[v+(D u)^{T} v\right]\right)\right)\right]^{T} v^{k} \\
& +(D F(u, p))^{T} v^{k} \quad \text { weakly in } L_{\text {loc }}^{r}(\Omega)^{2} .
\end{aligned}
$$

This combined with (3.40) thus yields

$$
\begin{aligned}
\sigma^{k}= & M^{k} u+\left[D P\left(v+(D u)^{T} v-\nabla P\left(\operatorname{div}\left[v+(D u)^{T} v\right]\right)\right)\right]^{T} v^{k} \\
& +(D F(u, p))^{T} v^{k}
\end{aligned}
$$

Third step. Determination of the limit equation (3.8).

The function $u$ solves the equation (3.27) which, by (3.4) and similarly to (3.31), can read as

$$
u=P\left(v+(D u)^{T} v-\nabla P\left(\operatorname{div}\left[v+(D u)^{T} v\right]\right)\right)+F(u, p) .
$$

This implies that

$$
(D u)^{T} v^{k}=\left[D P\left(v+(D u)^{T} v-\nabla P\left(\operatorname{div}\left[v+(D u)^{T} v\right]\right)\right)\right]^{T} v_{k}+(D F(u, p))^{T} v^{k}
$$

Therefore, equating the previous equation with (3.42) yields

$$
\sigma^{k}=(D u)^{T} v^{k}+M^{k} u \quad \text { in } \Omega .
$$

It remains to pass to the limit as $k$ tends to infinity. Due to the equi-integrability of $v_{\varepsilon}$ in $L^{2}(\Omega)^{2}$ and by convergence (3.26) the sequence $\sigma^{k}$ strongly converges to 
$v+(D u)^{T} v$ in $L^{1}(\Omega)$. On the other hand, putting the function $w_{\varepsilon}^{\lambda, k}-w_{\varepsilon}^{\lambda}$ both in equations (3.7) and (3.38) we get the equality

$$
\int_{\Omega}\left|D w_{\varepsilon}^{\lambda, k}-D w_{\varepsilon}^{\lambda}\right|^{2} d x=\int_{\Omega}\left(D w_{\varepsilon}^{\lambda, k}-D w_{\varepsilon}^{\lambda}\right)^{T}\left(v_{\varepsilon}^{k}-v_{\varepsilon}-v^{k}+v\right) \cdot \lambda d x,
$$

which, again by the equi-integrability of $v_{\varepsilon}$, yields

$$
\lim _{k \rightarrow \infty} \sup _{\varepsilon>0}\left(\int_{\Omega}\left|D w_{\varepsilon}^{\lambda, k}-D w_{\varepsilon}^{\lambda}\right|^{2} d x\right)=0 .
$$

Estimate (3.44) implies that the sequence $M^{k}$ defined by (3.41) strongly converges in $L^{1}(\Omega)^{2 \times 2}$ to the matrix-valued function $M$ defined by (3.20). In particular, up to a subsequence $M^{k}$ converges to $M$ a.e. in $\Omega$. Then, by the Fatou lemma combined with (3.43) and the strong convergences of $\sigma^{k}$ in $L^{1}(\Omega)^{2}$ and $v_{k}$ in $L^{2}(\Omega)^{2}$, we get that the function $M u$ belongs to $L^{1}(\Omega)^{2}$. Finally, passing to the limit in (3.43) we obtain the equality

$$
v=M u \text { in } \Omega,
$$

which gives the limit equation (3.8).

Fourth step. Proof of inequality (3.19).

Similarly to (3.23) let $W_{\varepsilon}^{k}, k>0$, be the matrix-valued function defined by $W_{\varepsilon}^{k} \lambda:=$ $w_{\varepsilon}^{\lambda, k}$, where $w_{\varepsilon}^{\lambda, k}$ solves (3.38). We simply denote $w_{\varepsilon}^{i, k}$ when $\lambda=e_{i}:=(2-i, i-$ 1 ), for $i=1$, 2. Let $\varphi \in C_{c}^{1}(\Omega)^{2}$, and let $t \in \mathbb{R}$. Using (3.13) we have

$$
\begin{aligned}
\int_{\Omega}\left|D u_{\varepsilon}-D u-t D\left(W_{\varepsilon}^{k} \varphi\right)\right|^{2} d x= & \langle f, u\rangle_{H^{-1}(\Omega)^{2}, H_{0}^{1}(\Omega)^{2}}-\int_{\Omega}|D u|^{2} d x \\
& -2 t \int_{\Omega} D u_{\varepsilon}: D\left(W_{\varepsilon}^{k} \varphi\right) d x \\
& +t^{2} \int_{\Omega}\left|D\left(W_{\varepsilon}^{k} \varphi\right)\right|^{2} d x+o(1) .
\end{aligned}
$$

Moreover, similarly to the second convergences of (3.9) and (3.10), we have for $i, j=1,2$,

$$
\left\{\begin{array}{l}
D u_{\varepsilon}: D w_{\varepsilon}^{i, k} \longrightarrow M^{k} u \cdot e_{i} \\
D w_{\varepsilon}^{i, k} \cdot D w_{\varepsilon}^{j, k} \longrightarrow \hat{M}^{k} e_{i} \cdot e_{j},
\end{array} \quad \text { weakly in } L_{\mathrm{loc}}^{s}(\Omega), \text { for any } s \in[1,2),\right.
$$

where (compare to the definition (3.41) of $M^{k}$ ) the matrix-valued $\hat{M}^{k}$ is defined by

$$
\left(D w_{\varepsilon}^{i, k}\right)^{T} v_{\varepsilon}^{k} \longrightarrow \hat{M}^{k} e_{i} \quad \text { weakly in } L^{2}(\Omega)^{2} .
$$


Then, from convergences (3.39) and (3.46) we deduce that

$$
\begin{aligned}
& \int_{\Omega} D u_{\varepsilon}: D\left(W_{\varepsilon}^{k} \varphi\right) d x=\sum_{i=1}^{2} \int_{\Omega} D u_{\varepsilon}: D w_{\varepsilon}^{i, k} \varphi_{i} d x+o(1) \underset{\varepsilon \rightarrow 0}{\longrightarrow} \int_{\Omega} M^{k} u \cdot \varphi d x, \\
& \int_{\Omega}\left|D\left(W_{\varepsilon}^{k} \varphi\right)\right|^{2} d x=\sum_{i, j=1}^{2} \int_{\Omega} D w_{\varepsilon}^{i, k}: D w_{\varepsilon}^{j, k} \varphi_{i} \varphi_{j} d x+o(1) \underset{\varepsilon \rightarrow 0}{\longrightarrow} \int_{\Omega} \hat{M}^{k} \varphi \cdot \varphi d x .
\end{aligned}
$$

This combined with (3.45) implies that

$$
\begin{aligned}
\int_{\Omega}\left|D u_{\varepsilon}-D u-t D\left(W_{\varepsilon}^{k} \varphi\right)\right|^{2} d x= & \langle f, u\rangle_{H^{-1}(\Omega)^{2}, H_{0}^{1}(\Omega)^{2}}-\int_{\Omega}|D u|^{2} d x \\
& -2 t \int_{\Omega} M^{k} u \cdot \varphi d x \\
& +t^{2} \int_{\Omega} \hat{M}^{k} \varphi \cdot \varphi d x+o(1) .
\end{aligned}
$$

Therefore, we have for any $t \in \mathbb{R}$,

$t^{2} \int_{\Omega} \hat{M}^{k} \varphi \cdot \varphi d x-2 t \int_{\Omega} M^{k} u \cdot \varphi d x+\langle f, u\rangle_{H^{-1}(\Omega)^{2}, H_{0}^{1}(\Omega)^{2}}-\int_{\Omega}|D u|^{2} d x \geq 0$,

hence

$$
\left(\int_{\Omega} M^{k} u \cdot \varphi d x\right)^{2} \leq\left(\langle f, u\rangle_{H^{-1}(\Omega)^{2}, H_{0}^{1}(\Omega)^{2}}-\int_{\Omega}|D u|^{2} d x\right) \int_{\Omega} \hat{M}^{k} \varphi \cdot \varphi d x .
$$

Let $\delta>0$, and let $\omega$ be an open set such that $\omega \Subset \Omega$. Since by (3.41) and (3.47) $M^{k}$ and $\hat{M}^{k}$ belong to $L^{s}(\omega)^{2 \times 2}$ for $s \in[1,2)$, putting in (3.49) strong approximations $\varphi$ of $\frac{1_{\omega} u}{1+\delta|u|}$ in $L^{2 s^{\prime}}(\Omega)^{2}$, we get

$$
\begin{aligned}
\left(\int_{\omega} \frac{M^{k} u \cdot u}{1+\delta|u|} d x\right)^{2} & \leq\left(\langle f, u\rangle_{H^{-1}(\Omega)^{2}, H_{0}^{1}(\Omega)^{2}}-\int_{\Omega}|D u|^{2} d x\right) \int_{\omega} \frac{\hat{M}^{k} u \cdot u}{(1+\delta|u|)^{2}} d x \\
& \leq\left(\langle f, u\rangle_{H^{-1}(\Omega)^{2}, H_{0}^{1}(\Omega)^{2}}-\int_{\Omega}|D u|^{2} d x\right) \int_{\Omega} \frac{\hat{M}^{k} u \cdot u}{(1+\delta|u|)^{2}} d x
\end{aligned}
$$

which by the arbitrariness of $\omega$ yields the inequality

$$
\left(\int_{\Omega} \frac{M^{k} u \cdot u}{1+\delta|u|} d x\right)^{2} \leq\left(\langle f, u\rangle_{H^{-1}(\Omega)^{2}, H_{0}^{1}(\Omega)^{2}}-\int_{\Omega}|D u|^{2} d x\right) \int_{\Omega} \frac{\hat{M}^{k} u \cdot u}{(1+\delta|u|)^{2}} d x .
$$

Recall that, by virtue of the equi-integrability of $v_{\varepsilon}$ in $L^{2}(\Omega)^{2}$, the sequences $M^{k}$ and $\hat{M}^{k}$ strongly converge to $M$ in $L^{1}(\Omega)^{2 \times 2}$, thus converge, up to a subsequence 
of $k$, a.e. in $\Omega$ and in a dominated way. Therefore, passing to the limit as $k \rightarrow \infty$ owing to the Fatou lemma for the left-hand side of (3.50) and owing to the Lebesgue dominated convergence theorem for the right-hand side of (3.50), it follows that

$$
\left(\int_{\Omega} \frac{M u \cdot u}{1+\delta|u|} d x\right)^{2} \leq\left(\langle f, u\rangle_{H^{-1}(\Omega)^{2}, H_{0}^{1}(\Omega)^{2}}-\int_{\Omega}|D u|^{2} d x\right) \int_{\Omega} \frac{M u \cdot u}{(1+\delta|u|)^{2}} d x<\infty
$$

which implies the inequality

$$
\int_{\Omega} \frac{M u \cdot u}{1+\delta|u|} d x \leq\langle f, u\rangle_{H^{-1}(\Omega)^{2}, H_{0}^{1}(\Omega)^{2}}-\int_{\Omega}|D u|^{2} d x .
$$

Finally, applying the Fatou lemma in (3.51) as $\delta \rightarrow 0$ we obtain the desired inequality (3.19).

Fifth step. Proof of equality (3.21) and of the corrector result (3.22).

Assume that $\Omega$ has a Lipschitz boundary, $v \in L^{r}(\Omega)^{N}$, with $r>2$, and $M \in$ $L^{m}(\Omega)^{2 \times 2}$, with $m>1$. Let $\varphi$ be a divergence free function in $C_{c}^{\infty}(\Omega)^{2}$. Putting $\varphi$ as test function in the limit Stokes equation (3.8) and using the representation formula (3.4) we have

$$
\begin{aligned}
\int_{\Omega} D u: D \varphi d x & +\int_{\Omega}(D u)^{T} v \cdot \varphi d x-\int_{\Omega}(v \otimes u): D \varphi d x+\int_{\Omega} M u \cdot \varphi d x \\
& =\langle f, u\rangle_{H^{-1}(\Omega)^{2}, H_{0}^{1}(\Omega)^{2}} .
\end{aligned}
$$

Due to the regularity of $\Omega$ the set of divergence free functions is known to be dense in the space of divergence free functions in $H_{0}^{1}(\Omega)^{2}$ (see, e.g., [18]). Moreover, by the higher integrability of $v$ and $M$ the mapping

$$
\varphi \longmapsto \int_{\Omega}(D u)^{T} v \cdot \varphi d x-\int_{\Omega}(v \otimes u): D \varphi d x+\int_{\Omega} M u \cdot \varphi d x
$$

is continuous in $H_{0}^{1}(\Omega)^{2}$. Therefore, considering in (3.52) a divergence free strong approximation $\varphi$ of $u$ in $H_{0}^{1}(\Omega)^{2}$ we get

$$
\begin{aligned}
\int_{\Omega}|D u|^{2} d x & +\int_{\Omega}(D u)^{T} v \cdot u d x-\int_{\Omega}(v \otimes u): D u d x+\int_{\Omega} M u \cdot u d x \\
& =\langle f, u\rangle_{H^{-1}(\Omega)^{2}, H_{0}^{1}(\Omega)^{2}}
\end{aligned}
$$

which is (3.21). This equality clearly implies the uniqueness of a solution $u \in$ $H_{0}^{1}(\Omega)^{2}$ of (3.8), with $M u \cdot u \in L^{1}(\Omega)$. 
It remains to prove the corrector result (3.22). Let $\varphi \in C_{c}^{\infty}(\Omega)$. Applying successively the triangle inequality and the Cauchy-Schwarz inequality we have

$$
\begin{aligned}
& \int_{\Omega}\left|D u_{\varepsilon}-D u-D\left(W_{\varepsilon} u\right)\right| d x \\
& \leq \int_{\Omega}\left|D u_{\varepsilon}-D u-D\left(W_{\varepsilon} \varphi\right)\right| d x+\int_{\Omega}\left|D\left(W_{\varepsilon}(u-\varphi)\right)\right| d x \\
& \leq \int_{\Omega}\left|D u_{\varepsilon}-D u-D\left(W_{\varepsilon} \varphi\right)\right| d x+\int_{\Omega}\left|D W_{\varepsilon}\right||u-\varphi| d x+\int_{\Omega}\left|W_{\varepsilon}\right||D u-D \varphi| d x \\
& \leq \int_{\Omega}\left|D u_{\varepsilon}-D u-D\left(W_{\varepsilon} \varphi\right)\right| d x+c\left\|W_{\varepsilon}\right\|_{H^{1}(\Omega)^{2 \times 2}}\|u-\varphi\|_{H_{0}^{1}(\Omega)^{2}},
\end{aligned}
$$

hence by the boundedness of $W_{\varepsilon}$ in $H_{0}^{1}(\Omega)^{2 \times 2}$,

$$
\int_{\Omega}\left|D u_{\varepsilon}-D u-D\left(W_{\varepsilon} u\right)\right| d x \leq \int_{\Omega}\left|D u_{\varepsilon}-D u-D\left(W_{\varepsilon} \varphi\right)\right| d x+c\|u-\varphi\|_{H_{0}^{1}(\Omega)^{2}} .
$$

On the other hand, proceeding as in fourth step owing to the second convergences of (3.20) and (3.10) (which hold in the weak-* sense of measures on $\Omega$ ) we get similarly to (3.48) the equality

$$
\begin{aligned}
\int_{\Omega}\left|D u_{\varepsilon}-D u-D\left(W_{\varepsilon} \varphi\right)\right|^{2} d x= & \langle f, u\rangle_{H^{-1}(\Omega)^{2}, H_{0}^{1}(\Omega)^{2}}-\int_{\Omega}|D u|^{2} d x \\
& -2 \int_{\Omega} M u \cdot \varphi d x+\int_{\Omega} M \varphi \cdot \varphi d x+o(1) .
\end{aligned}
$$

Hence, taking into account equality (3.21) and using the Hölder inequality combined with the embedding of $H_{0}^{1}(\Omega)$ in any $L^{s}(\Omega)$ space, it follows that

$$
\begin{aligned}
\int_{\Omega}\left|D u_{\varepsilon}-D u-D\left(W_{\varepsilon} \varphi\right)\right|^{2} d x & =\int_{\Omega} M(u-\varphi) \cdot(u-\varphi) d x+o(1) \\
& \leq c\|M\|_{L^{m}(\Omega)^{2 \times 2}}\|u-\varphi\|_{H_{0}^{1}(\Omega)^{2}}^{2}+o(1) .
\end{aligned}
$$

Therefore, by (3.53) and (3.54) we obtain the inequality

$\limsup _{\varepsilon \rightarrow 0} \int_{\Omega}\left|D u_{\varepsilon}-D u-D\left(W_{\varepsilon} u\right)\right| d x \leq c\|u-\varphi\|_{H_{0}^{1}(\Omega)^{2}}, \quad$ for any $\varphi \in C_{c}^{\infty}(\Omega)^{2}$,

which implies the desired convergence (3.22) and concludes the proof of Theorem 3.3.

As in the scalar case we show in the next section that the equi-integrability condition is crucial to derive the limit Brinkman equation (3.8) with the matrixvalued function $M$ introduced by L. Tartar $[15,17]$. 


\subsection{A counter-example}

Let $\Omega$ be a regular bounded domain of $\mathbb{R}^{2}$. For $\varepsilon>0$, let $\omega_{\varepsilon}$ be the intersection of $\Omega$ with the periodic lattice of disks of center $2 \varepsilon \kappa, \kappa \in \mathbb{Z}^{2}$, and of radius $\varepsilon r_{\varepsilon}$ such that

$$
\frac{4 \pi}{\varepsilon^{2}\left|\ln r_{\varepsilon}\right|} \underset{\varepsilon \rightarrow 0}{\longrightarrow} \gamma \in(0, \infty) \text {. }
$$

This geometry was used by Cioranescu, Murat [6] for the Laplace equation and by Allaire [2] for the Stokes equation, in order to derive a "strange term" of zero-order from the homogenization of the Dirichlet boundary conditions on the small disks.

In the square $Y:=(-1,1)^{2}$, let $Q$ be the disk centered at the origin and of radius 1, and let $Q_{r_{\varepsilon}}$ be the disk of same center and of radius $r_{\varepsilon}$ with measure $\left|Q_{r_{\varepsilon}}\right|=\pi r_{\varepsilon}^{2}$. Then, for $f \in H^{-1}(\Omega)^{2}$, we consider the Stokes equation

$$
\begin{cases}-\Delta u_{\varepsilon}+\frac{1_{\omega_{\varepsilon}}}{\left|Q_{r_{\varepsilon}}\right|} J u_{\varepsilon}+\nabla p_{\varepsilon}=f & \text { in } \Omega \\ \operatorname{div}\left(u_{\varepsilon}\right)=0 & \text { in } \Omega \\ u_{\varepsilon}=0 & \text { on } \partial \Omega .\end{cases}
$$

Note that, in view of the definition of $\omega_{\varepsilon}$, we have $\left|\omega_{\varepsilon}\right| \approx|\Omega|\left|Q_{r_{\varepsilon}}\right|$. Moreover, if $z_{\varepsilon} \in H_{0}^{1}(\Omega)$ is the solution of the Laplace equation

$$
\Delta z_{\varepsilon}=\frac{1_{\omega_{\varepsilon}}}{\left|Q_{r_{\varepsilon}}\right|} \text { in } \mathcal{D}^{\prime}(\Omega)
$$

we have

$$
\frac{1_{\omega_{\varepsilon}}}{\left|Q_{r_{\varepsilon}}\right|}=\operatorname{curl}\left(v_{\varepsilon}\right) \text { in } \mathcal{D}^{\prime}(\Omega), \quad \text { where } \quad v_{\varepsilon}:=J \nabla z_{\varepsilon} .
$$

Hence, the Stokes problem (3.57) is of the same type as (3.6). On the other hand, using successively the Cauchy-Schwarz inequality and the estimate (3.67) below combined with (3.56) we have

$$
\int_{\Omega}\left|\nabla z_{\varepsilon}\right|^{2} d x=-\frac{\left|\omega_{\varepsilon}\right|}{\left|Q_{r_{\varepsilon}}\right|} f_{\omega_{\varepsilon}} z_{\varepsilon} d x \leq \frac{\left|\omega_{\varepsilon}\right|}{\left|Q_{r_{\varepsilon}}\right|}\left(f_{\omega_{\varepsilon}} z_{\varepsilon}^{2} d x\right)^{\frac{1}{2}} \leq c\left\|\nabla z_{\varepsilon}\right\|_{L^{2}(\Omega)^{2}},
$$

which implies that $z_{\varepsilon}$ is bounded in $H_{0}^{1}(\Omega)$. Therefore, the sequence $v_{\varepsilon}$ is bounded in $L^{2}(\Omega)^{2}$. Moreover, since by periodicity the sequence $\frac{1_{\omega_{\varepsilon}}}{\left|Q_{r_{\varepsilon}}\right|}$ converges weakly-* to $\frac{1}{4}$ in $\mathcal{M}(\Omega)$, we get

$$
v_{\varepsilon} \longrightarrow v \text { weakly in } L^{2}(\Omega)^{2}, \quad \text { with } \operatorname{curl}(v)=\frac{1}{4} \text { in } \mathcal{D}^{\prime}(\Omega) .
$$

On the other hand, it is not difficult to check that $v_{\varepsilon}$ is not equi-integrable in $L^{2}(\Omega)^{2}$. In fact, the following result shows that Theorem 3.3 does not hold for this particular sequence $v_{\varepsilon}$ : 
Theorem 3.5. The sequence $u_{\varepsilon}$ weakly converges in $H_{0}^{1}(\Omega)^{2}$ to the solution $u$ of the Brinkman equation

$$
\begin{cases}-\Delta u+\frac{1}{4} J u+\nabla p+\Gamma u=f & \text { in } \Omega \\ \operatorname{div}(u)=0 & \text { in } \Omega \\ u=0 & \text { on } \partial \Omega\end{cases}
$$

where the extra zero-order term $\Gamma u$ is given by

$$
\left(\frac{1_{\omega_{\varepsilon}}}{\left|Q_{r_{\varepsilon}}\right|}-\frac{1}{4}\right) J u_{\varepsilon} \longrightarrow \Gamma u \quad \text { weakly-* in } \mathcal{M}(\Omega)^{2},
$$

and $\Gamma$ is the constant matrix defined by

$$
\Gamma:=\frac{1}{4\left(\gamma^{2}+1\right)}(\gamma I-J) .
$$

Moreover, the matrix obtained from convergence (3.20) according to the Tartar approach is given by

$$
M=\frac{1}{4 \gamma} I .
$$

Remark 3.6. The matrix $\Gamma$ of the Brinkman equation (3.61) is not symmetric contrary to the matrix $M$ arising in the Tartar approach. Moreover, we have

$$
\Gamma u \cdot u<M u \cdot u \text { if } u \neq 0 \text {. }
$$

The gap between the two previous energies (which are the energies dissipated by viscosity according to [14]) is due to the loss of equi-integrability of the sequence $v_{\varepsilon}$ defined by (3.59). Therefore, the equi-integrability of $v_{\varepsilon}$ can be regarded as the best condition to ensure the result of Theorem 3.3.

Remark 3.7. It is worth to mention that the pathology displayed in Theorem 3.5 is not due to the absence of correctors. Indeed, with the oscillating sequences $v_{\varepsilon}^{1}, v_{\varepsilon}^{2}$ defined by (3.68), (3.69) below, the following corrector result holds:

Proposition 3.8. Assume that $u \in W^{1, r}(\Omega)^{2}$ for some $r>2$. Then, we have

$$
\begin{array}{r}
u_{\varepsilon}-u-v_{1} v_{\varepsilon}^{1}-v_{2} v_{\varepsilon}^{2} \longrightarrow 0 \text { strongly in } H^{1}(\Omega), \\
\text { where } v=\left(v_{1}, v_{2}\right):=\frac{1}{\gamma^{2}+1}\left(-u_{1}+\gamma u_{2},-u_{2}-\gamma u_{1}\right) .
\end{array}
$$

Remark 3.9. If the right-hand side $f$ belongs to $W^{-1, r}(\Omega)^{2}$ for some $r>2$, then using the regularity results for the Stokes equation (see, e.g., [10]) the solution $u$ of the Stokes equation (3.61) belongs to $W^{1, r}(\Omega)^{2}$. This provides a quite general condition under which the strong convergence (3.65) holds. 
The proof of Theorem 3.5 is partially based on the properties of the test functions $v_{\varepsilon}^{1}, v_{\varepsilon}^{2}$ defined by (3.68), (3.69) below, and introduced by Allaire [2]. They were also used in [5] to derive a homogenized Brinkman type equation but, contrary to (3.6), from a Stokes equation without zero-order term. More precisely, in [2] the velocity is assumed to be zero in the set $\omega_{\varepsilon}$. In [5] the viscosity is assumed to be very high in cylinders of section $\omega_{\varepsilon}$, which leads to a three-dimensional nonlocal Brinkman equation. In the perturbed Stokes equation (3.57) a highly oscillating zero-order term is concentrated on $\omega_{\varepsilon}$.

On the one hand, the sets $Q_{r_{\varepsilon}}$ and $\omega_{\varepsilon}$ satisfy the following estimates:

Lemma 3.10. There exists a constant $C>0$ such that

$$
\begin{gathered}
\forall V \in H^{1}(Y), \quad\left|f_{Q_{r_{\varepsilon}}} V d y-f_{Y} V d y\right| \leq C \sqrt{\left|\ln r_{\varepsilon}\right|}\|\nabla V\|_{L^{2}(Y)^{2}}, \\
\forall v \in H_{0}^{1}(\Omega), \quad f_{\omega_{\varepsilon}}|v|^{2} d x \leq C\left(1+\varepsilon^{2}\left|\ln r_{\varepsilon}\right|\right)\|\nabla v\|_{L^{2}(\Omega)^{2}}^{2} .
\end{gathered}
$$

Proof. Estimate (3.66) can be easily proved using the polar coordinates. Estimate (3.67) is an immediate consequence of the Lemma 3 of [12], and can also be deduced from (3.66).

On the other hand, consider the $\varepsilon Y$-periodic functions $v_{\varepsilon}^{i}$ and $p_{\varepsilon}^{i}$, for $i=1,2$, defined by

$$
v_{\varepsilon}^{i}(x):=V_{\varepsilon}^{i}\left(\frac{x}{\varepsilon}\right), \quad p_{\varepsilon}^{i}(x):=\frac{1}{\varepsilon} P_{\varepsilon}^{i}\left(\frac{x}{\varepsilon}\right), \quad \text { for } x \in \mathbb{R}^{2},
$$

where $V_{\varepsilon}^{i} \in H_{\#}^{1}(Y)$ are $P_{\varepsilon}^{i} \in L^{2}(Y)$ are the $Y$-periodic functions defined by

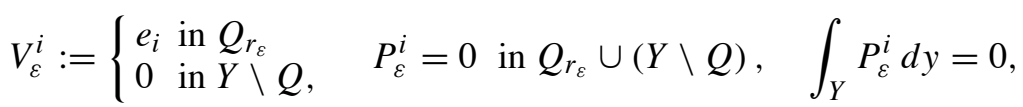

which solve the Stokes equation

$$
-\Delta V_{\varepsilon}^{i}+\nabla P_{\varepsilon}^{i}=0 \quad \text { in } Q_{r_{\varepsilon}} \backslash \bar{Q}
$$

Moreover, the sequences $V_{\varepsilon}^{i}$ and $P_{\varepsilon}^{i}$ satisfy the following estimates:

Lemma 3.11. There exists a constant $C>0$ such that

$$
\left\{\begin{array}{l}
\left\|V_{\varepsilon}^{i}\right\|_{L^{2}(Y)^{2}}+\left\|D V_{\varepsilon}^{i}\right\|_{L^{2}(Y)^{2 \times 2}}^{2}+\left\|P_{\varepsilon}^{i}\right\|_{L^{2}(Y)}^{2} \leq \frac{C}{\left|\ln r_{\varepsilon}\right|} \\
\left\|V_{\varepsilon}^{i}\right\|_{L^{\infty}(Y)^{2}} \leq C
\end{array}\right.
$$


and for any function $V \in H^{1}(Y)$,

$$
\begin{aligned}
& \left|\int_{Y} D V_{\varepsilon}^{i}: D V d y-\int_{Y} P_{\varepsilon}^{i} \operatorname{div}(V) d y-\gamma_{\varepsilon}^{i} e_{i} \cdot\left(f_{Q_{r_{\varepsilon}}} V-f_{Y \backslash Q} V\right)\right| \\
& \quad \leq \frac{C}{\left|\ln r_{\varepsilon}\right|}\|D V\|_{L^{2}(Y)^{2 \times 2}}
\end{aligned}
$$

where $f$ denotes the average value and

$$
\gamma_{\varepsilon}^{i} \underset{\varepsilon \rightarrow 0}{\approx} \frac{4 \pi}{\left|\ln r_{\varepsilon}\right|} .
$$

Proof. Estimate (3.71) can be proved using the polar coordinates (see also [2]). Estimate (3.72) is a straightforward consequence of the Lemma 3.3 of [4] (with a refinement for the right-hand side of the inequality).

Proof of Theorem 3.5. The proof is divided into two steps. In the first step we determine the homogenized Brinkman equation (3.61). The second step is devoted to the computation of the matrix $M$ defined in the Tartar approach.

First step. Determination of the homogenized equation.

Using $u_{\varepsilon}$ as test function we have

$$
\int_{\Omega}\left|D u_{\varepsilon}\right|^{2}=\left\langle f, u_{\varepsilon}\right\rangle_{H^{-1}(\Omega)^{2}, H_{0}^{1}(\Omega)^{2}} \leq c\|f\|_{H^{-1}(\Omega)^{2}}\left\|D u_{\varepsilon}\right\|_{L^{2}(\Omega)^{2 \times 2}}
$$

which implies that $u_{\varepsilon}$ is bounded in $H_{0}^{1}(\Omega)^{2}$. On the other hand, let $\varphi \in C_{c}^{\infty}(\Omega)$ with zero $\Omega$-average. There exists (see, e.g., [3]) a vector-valued function $\Phi \in$ $C_{c}^{\infty}(\Omega)^{2}$ such that

$$
\operatorname{div}(\Phi)=\varphi \text { in } \Omega \quad \text { and } \quad\|\Phi\|_{H_{0}^{1}(\Omega)^{2}} \leq c\|\varphi\|_{L^{2}(\Omega)}
$$

where the constant $c$ is independent of $\varphi, \Phi$. Using $\Phi$ as test function in equation (3.57) and applying successively the Cauchy-Schwarz inequality, estimates (3.67) and (3.56) we get

$$
\begin{aligned}
& \left|\int_{\Omega} p_{\varepsilon} \varphi d x\right| \leq\left|\langle f, \Phi\rangle_{H^{-1}(\Omega)^{2}, H_{0}^{1}(\Omega)^{2}}\right|+\left|\int_{\Omega} D u_{\varepsilon}: D \Phi\right|+\left|\int_{\Omega} \frac{1_{\omega_{\varepsilon}}}{\left|Q_{r_{\varepsilon}}\right|} J u_{\varepsilon} \cdot \Phi\right| \\
& \leq c\|D \Phi\|_{L^{2}(\Omega)^{2 \times 2}}+c\left(f_{\omega_{\varepsilon}}\left|u_{\varepsilon}\right|^{2}\right)^{\frac{1}{2}}\left(f_{\omega_{\varepsilon}}|\Phi|^{2}\right)^{\frac{1}{2}} \\
& \leq c\|D \Phi\|_{L^{2}(\Omega)^{2 \times 2}}+c \varepsilon^{2}\left|\ln r_{\varepsilon}\right|\left\|D u_{\varepsilon}\right\|_{L^{2}(\Omega)^{2 \times 2}}\|D \Phi\|_{L^{2}(\Omega)^{2 \times 2}} \\
& \leq c\|\varphi\|_{L^{2}(\Omega)} \text {. }
\end{aligned}
$$


This combined with the regularity of $\Omega$ implies that $p_{\varepsilon}$ is bounded in $L^{2}(\Omega) / \mathbb{R}$. Therefore, up to a subsequence the following convergences hold

$$
\left\{\begin{array}{l}
u_{\varepsilon} \longrightarrow u \text { weakly in } H_{0}^{1}(\Omega)^{2} \\
p_{\varepsilon} \longrightarrow p \text { weakly in } L^{2}(\Omega) / \mathbb{R} .
\end{array}\right.
$$

Now, we have to determine the limit of the sequence $\frac{1_{\omega_{\varepsilon}}}{\left|Q_{r_{\varepsilon}}\right|} J u_{\varepsilon}$. On the one hand, re-scaling inequality (3.72) we obtain that the functions $v_{\varepsilon}^{i}$ and $p_{\varepsilon}^{i}, i=1,2$, of (3.68) and any function $v \in H_{0}^{1}(\Omega)^{2}$ satisfy the inequality

$$
\begin{aligned}
& \left|\int_{\Omega} D v_{\varepsilon}^{i}: D v-\int_{\Omega} p_{\varepsilon}^{i} \operatorname{div}(v)-\frac{\gamma_{\varepsilon}^{i}}{\varepsilon^{2}} e_{i} \cdot\left(\int_{\Omega} \frac{1_{\omega_{\varepsilon}}}{\left|Q_{r_{\varepsilon}}\right|} v-\int_{\Omega} \frac{1_{Y \backslash Q}}{|Y \backslash Q|}\left(\frac{x}{\varepsilon}\right) v\right)\right| \\
& \quad \leq \frac{c}{\varepsilon\left|\ln r_{\varepsilon}\right|}\|D v\|_{L^{2}(\Omega)^{2 \times 2} .}
\end{aligned}
$$

Moreover, by (3.71) and (3.56) the following convergences hold

$$
\left\{\begin{array}{l}
v_{\varepsilon}^{i} \longrightarrow 0 \text { weakly in } H^{1}(\Omega)^{2} \\
p_{\varepsilon}^{i} \longrightarrow 0 \text { weakly in } L^{2}(\Omega) / \mathbb{R} .
\end{array}\right.
$$

Then, applying inequality (3.75) with $v=\varphi u_{\varepsilon}, \varphi \in C_{c}^{\infty}(\Omega)$, we deduce from (3.73) and (3.56) that

$$
\int_{\Omega} D v_{\varepsilon}^{i}: D u_{\varepsilon} \varphi-(\gamma+o(1)) e_{i} \cdot\left(\int_{\Omega} \frac{1_{\omega_{\varepsilon}}}{\left|Q_{r_{\varepsilon}}\right|} \varphi u_{\varepsilon}-\int_{\Omega} \frac{1}{4} \varphi u\right)=o(1) .
$$

On the other hand, putting $\varphi v_{\varepsilon}^{i}$ as test function in (3.57), using that $v_{\varepsilon}^{i}=e_{i}$ in $\omega_{\varepsilon}$ and the convergences (3.76), (3.74), we have

$$
\int_{\Omega} D u_{\varepsilon}: D v_{\varepsilon}^{i} \varphi+\int_{\Omega} \frac{1_{\omega_{\varepsilon}}}{\left|Q_{r_{\varepsilon}}\right|} J u_{\varepsilon} \cdot e_{i} \varphi=o(1)
$$

Denote

$$
v:=\lim _{\varepsilon \rightarrow 0} \frac{1_{\omega_{\varepsilon}}}{\left|Q_{r_{\varepsilon}}\right|} J u_{\varepsilon} \quad \text { weakly-* in } \mathcal{M}(\Omega)^{2},
$$

where the limit holds up to a subsequence by virtue of the estimate (3.67) combined with the Cauchy-Schwarz inequality. Then, equating (3.77) and (3.78) and passing to the limit we get for $i=1,2$,

$$
\int_{\Omega} \varphi e_{i} \cdot v=\gamma \int_{\Omega} \varphi e_{i} \cdot J v+\frac{\gamma}{4} \int_{\Omega} \varphi e_{i} \cdot u, \quad \text { for any } \varphi \in C_{c}^{\infty}(\Omega),
$$

which implies the equality $v=\gamma J v+\frac{\gamma}{4} u$. Hence, we deduce the convergence $\frac{1_{\omega_{\varepsilon}}}{\left|Q_{r_{\varepsilon}}\right|} J u_{\varepsilon} \longrightarrow v=\frac{\gamma}{4}(I-\gamma J)^{-1} u=\frac{\gamma}{4\left(\gamma^{2}+1\right)}(I+\gamma J) u \quad$ weakly-* in $\mathcal{M}(\Omega)^{2}$. 
Therefore, passing to the limit in (3.57) with (3.79) we obtain the homogenized equation

$$
-\Delta u+\frac{1}{4} J u+\nabla p+\frac{1}{4\left(\gamma^{2}+1\right)}(\gamma I-J) u=f \quad \text { in } \mathcal{D}^{\prime}(\Omega),
$$

which yields the desired Brinkman equation (3.61) with the matrix $\Gamma$ of (3.63).

Second step. Derivation of the matrix $M$.

Let $\lambda \in \mathbb{R}^{2}$. Consider the solutions $W_{\sharp, \varepsilon}^{\lambda} \in H_{\sharp}^{1}(Y)$ (the set of the $Y$-periodic functions in $H_{\text {loc }}^{1}\left(\mathbb{R}^{2}\right)$ ) and $Q_{\sharp, \varepsilon}^{\lambda} \in L_{\sharp}^{2}(Y) / \mathbb{R}$ of the perturbed Stokes problem

$$
\begin{cases}-\Delta W_{\sharp, \varepsilon}^{\lambda}+\varepsilon\left(\frac{1 Q_{r_{\varepsilon}}}{\left|Q_{r_{\varepsilon}}\right|}-\frac{1}{4}\right) J \lambda+\nabla Q_{\sharp, \varepsilon}^{\lambda}=0 & \text { in } \mathbb{R}^{2} \\ \operatorname{div}\left(W_{\sharp, \varepsilon}^{\lambda}\right)=0 & \text { in } \mathbb{R}^{2} \\ W_{\sharp, \varepsilon}^{\lambda} & \text { is } Y \text {-periodic } \\ \int_{Y} W_{\sharp, \varepsilon}^{\lambda}=0 . & \end{cases}
$$

Note that the first equation of (3.81) is equivalent to the variational formulation in the torus,

$\forall V \in H_{\sharp}^{1}(Y), \int_{Y} D W_{\sharp, \varepsilon}^{\lambda}: D V d y+\varepsilon\left(f_{Q_{r_{\varepsilon}}} V-f_{Y} V\right) \cdot J \lambda-\int_{Y} Q_{\sharp, \varepsilon}^{\lambda} \operatorname{div}(V) d y=0$.

Hence, the re-scaled functions $w_{\sharp, \varepsilon}^{\lambda}$ and $q_{\sharp, \varepsilon}^{\lambda}$ defined by

$$
w_{\sharp, \varepsilon}^{\lambda}(x):=\varepsilon W_{\sharp, \varepsilon}^{\lambda}\left(\frac{x}{\varepsilon}\right) \quad \text { and } \quad q_{\sharp, \varepsilon}^{\lambda}(x):=Q_{\sharp, \varepsilon}^{\lambda}\left(\frac{x}{\varepsilon}\right), \quad \text { for } x \in \Omega \text {, }
$$

are $\varepsilon Y$-periodic solutions of the problem

$$
\begin{cases}-\Delta w_{\sharp, \varepsilon}^{\lambda}+\left(\frac{1 Q_{r_{\varepsilon}}}{\left|Q_{r_{\varepsilon}}\right|}\left(\frac{x}{\varepsilon}\right)-\frac{1}{4}\right) J \lambda+\nabla q_{\sharp, \varepsilon}^{\lambda}=0 & \text { in } \mathbb{R}^{2} \\ \operatorname{div}\left(w_{\sharp, \varepsilon}^{\lambda}\right)=0 & \text { in } \mathbb{R}^{2} .\end{cases}
$$

First of all, let us determine a priori estimates satisfied by the sequences $W_{\sharp, \varepsilon}^{\lambda}, w_{\sharp, \varepsilon}^{\lambda}$, $Q_{\sharp, \varepsilon}^{\lambda}$, and $q_{\sharp, \varepsilon}^{\lambda}$. Putting $W_{\sharp, \varepsilon}^{\lambda}$ as test function in equation (3.82) we have

$$
\int_{Y}\left|D W_{\sharp, \varepsilon}^{\lambda}\right|^{2} d y+\varepsilon f_{Q_{r_{\varepsilon}}} J \lambda \cdot W_{\sharp, \varepsilon}^{\lambda} d y=0,
$$


hence by the estimates (3.66) of Lemma 3.10 and (3.56)

$$
\begin{aligned}
\left\|D W_{\sharp, \varepsilon}^{\lambda}\right\|_{L^{2}(Y)^{2 \times 2}}^{2} & =\varepsilon\left|f_{Q_{r_{\varepsilon}}} W_{\sharp, \varepsilon}^{\lambda} d y\right| \\
& \leq C \varepsilon \sqrt{\left|\ln r_{\varepsilon}\right|}\left\|D W_{\sharp, \varepsilon}^{\lambda}\right\|_{L^{2}(Y)^{2 \times 2}} \\
& \leq c\left\|D W_{\sharp, \varepsilon}^{\lambda}\right\|_{L^{2}(Y)^{2 \times 2}} .
\end{aligned}
$$

Therefore, $W_{\sharp, \varepsilon}^{\lambda}$ is bounded in $H_{\sharp}^{1}(Y)^{2}$, and there exists a constant vector $\bar{W}^{\lambda} \in \mathbb{R}^{2}$ such that up to a subsequence we have

$$
\lim _{\varepsilon \rightarrow 0}\left(\varepsilon f_{Q_{r_{\varepsilon}}} W_{\sharp, \varepsilon}^{\lambda} d y\right)=\bar{W}^{\lambda} .
$$

On the other hand, let $\varphi \in C_{\sharp}^{\infty}(Y)$ with zero $Y$-average. There exists $\Phi \in C_{\sharp}^{\infty}(Y)^{2}$ with zero $Y$-average such that

$$
\operatorname{div}(\Phi)=\varphi \text { in } \mathbb{R}^{2} \text { and }\|\Phi\|_{L^{2}(Y)^{2 \times 2}} \leq c\|\varphi\|_{L^{2}(Y)},
$$

where $c$ is a constant independent of $\varphi, \Phi$. Putting $\Phi$ as test function in (3.82) we have by (3.66) and (3.56)

$$
\begin{aligned}
\left|\int_{Y} Q_{\sharp, \varepsilon}^{\lambda} \varphi d y\right| & \leq\left|\int_{Y} D W_{\sharp, \varepsilon}^{\lambda}: D \Phi d y\right|+\varepsilon\left|f_{Q_{r_{\varepsilon}}} \Phi d y\right| \\
& \leq c\|D \Phi\|_{L^{2}(Y)^{2 \times 2}}+c \varepsilon \sqrt{\left|\ln r_{\varepsilon}\right|}\|D \Phi\|_{L^{2}(Y)^{2 \times 2}} \\
& \leq c\|D \Phi\|_{L^{2}(Y)^{2 \times 2}} \leq c\|\varphi\|_{L^{2}(Y)},
\end{aligned}
$$

hence $Q_{\sharp, \varepsilon}^{\lambda}$ is bounded in $L_{\sharp}^{2}(Y) / \mathbb{R}$. From the boundedness and the $Y$-periodicity of $W_{\sharp, \varepsilon}^{\lambda}$ and $Q_{\sharp, \varepsilon}^{\lambda}$ we thus deduce that the sequences $w_{\sharp, \varepsilon}^{\lambda}$ and $q_{\sharp, \varepsilon}^{\lambda}$ of (3.83) satisfy the convergences

$$
\begin{cases}w_{\sharp, \varepsilon}^{\lambda} \longrightarrow 0 & \text { weakly in } H^{1}(\Omega)^{2} \\ q_{\sharp, \varepsilon}^{\lambda} \longrightarrow 0 & \text { weakly in } L^{2}(\Omega) / \mathbb{R} .\end{cases}
$$

Now, let us check that the periodic function $w_{\sharp, \varepsilon}^{\lambda}$ of (3.84) gives the same matrix $M$ (3.20) as the function $w_{\varepsilon}^{\lambda}$ of (3.7) which satisfies a Dirichlet boundary condition. Since $M$ is symmetric, this is equivalent to prove that for any $\lambda \in \mathbb{R}^{2}$,

$$
\left(D w_{\sharp, \varepsilon}^{\lambda}\right)^{T} v_{\varepsilon} \cdot \lambda-\left(D w_{\varepsilon}^{\lambda}\right)^{T} v_{\varepsilon} \cdot \lambda \longrightarrow 0 \quad \text { in } \mathcal{D}^{\prime}(\Omega),
$$

where $v_{\varepsilon}$ is defined by (3.59). Let $\varphi \in C_{c}^{\infty}(\Omega)$. Putting $\varphi w_{\sharp, \varepsilon}^{\lambda}$ in the equation (3.7) satisfied by $w_{\varepsilon}^{\lambda}$ and $\varphi w_{\varepsilon}^{\lambda}$ in the equation (3.84) satisfied by $w_{\sharp, \varepsilon}^{\lambda}$, and using 
the convergences (3.87) satisfied by $w_{\sharp, \varepsilon}^{\lambda}, q_{\sharp, \varepsilon}^{\lambda}$ as well as the similar ones satisfied by $w_{\varepsilon}^{\lambda}, q_{\varepsilon}^{\lambda}$, we get

$$
\left\{\begin{array}{l}
\int_{\Omega} D w_{\varepsilon}^{\lambda}: D w_{\sharp, \varepsilon}^{\lambda} \varphi-\int_{\Omega} \operatorname{curl}\left(v_{\varepsilon}\right) J w_{\sharp, \varepsilon}^{\lambda} \cdot \lambda \varphi \underset{\varepsilon \rightarrow 0}{\longrightarrow} 0 \\
\int_{\Omega} D w_{\sharp, \varepsilon}^{\lambda}: D w_{\varepsilon}^{\lambda} \varphi-\int_{\Omega} \operatorname{curl}\left(v_{\varepsilon}\right) J w_{\varepsilon}^{\lambda} \cdot \lambda \varphi \underset{\varepsilon \rightarrow 0}{\longrightarrow} 0 .
\end{array}\right.
$$

Moreover, by the representation formula (3.11) we have

$$
\left\{\begin{array}{l}
\operatorname{curl}\left(v_{\varepsilon}\right) J w_{\sharp, \varepsilon}^{\lambda}-\left(D w_{\sharp, \varepsilon}^{\lambda}\right)^{T} v_{\varepsilon} \longrightarrow 0 \\
\operatorname{curl}\left(v_{\varepsilon}\right) J w_{\varepsilon}^{\lambda}-\left(D w_{\varepsilon}^{\lambda}\right)^{T} v_{\varepsilon} \longrightarrow 0
\end{array} \quad \text { in } \mathcal{D}^{\prime}(\Omega)^{2} .\right.
$$

Therefore, combining (3.89) and (3.90) we obtain the desired convergence (3.88).

It remains to determine the matrix $M$. On the one side putting $w_{\sharp, \varepsilon}^{\lambda}$ as test function in (3.84) and using the convergences (3.88), (3.20), and on the other side using the $\varepsilon Y$-periodicity of $w_{\sharp, \varepsilon}^{\lambda}(3.83)$, we get similarly to (3.9) and up to a subsequence

$$
\left|D w_{\sharp, \varepsilon}^{\lambda}\right|^{2} \longrightarrow M \lambda \cdot \lambda \text { and }\left|D w_{\sharp, \varepsilon}^{\lambda}\right|^{2} \longrightarrow \lim _{\varepsilon \rightarrow 0}\left(f_{Y}\left|D W_{\sharp, \varepsilon}^{\lambda}\right|^{2} d y\right) \text { weakly-* in } \mathcal{M}(\Omega) \text {. }
$$

This combined with (3.85) and (3.86) gives

$$
M \lambda \cdot \lambda=\frac{1}{4} J \bar{W}^{\lambda} \cdot \lambda
$$

Let us compute the constant vector $\bar{W}^{\lambda}$. To this end, putting the divergence free function $W_{\sharp, \varepsilon}^{\lambda}$ in the inequality (3.72) satisfied by $V_{\varepsilon}^{i}, i=1,2$, and taking into account the estimates (3.71), (3.56) and the boundedness of $W_{\sharp, \varepsilon}^{\lambda}$ in $H^{1}(Y)^{2}$, we have

$$
\int_{Y} D V_{\varepsilon}^{i}: D W_{\sharp, \varepsilon}^{\lambda} d y=\gamma_{\varepsilon}^{i} e_{i} \cdot\left(f_{Q_{r_{\varepsilon}}} W_{\sharp, \varepsilon}^{\lambda} d y\right)+o(\varepsilon) .
$$

Moreover, putting the divergence free function $V_{\varepsilon}^{i}$ in (3.82) with $V_{\varepsilon}^{i}=e_{i}$ in $Q_{r_{\varepsilon}}$, we get

$$
\int_{Y} D W_{\sharp, \varepsilon}^{\lambda}: D V_{\varepsilon}^{i} d y=-\varepsilon\left(f_{Q_{r_{\varepsilon}}} V_{\varepsilon}^{i}-f_{Y} V_{\varepsilon}^{i}\right) \cdot J \lambda=\varepsilon J e_{i} \cdot \lambda+o(\varepsilon),
$$

since by (3.71) $V_{\varepsilon}^{i}$ strongly converges to zero in $L^{2}(Y)^{2}$. The estimates (3.93) and (3.94) divided by $\varepsilon$ together with (3.86), (3.73) and (3.56) imply that

$$
\gamma e_{i} \cdot \bar{W}^{\lambda}=J e_{i} \cdot \lambda \quad \text { or equivalently } \quad \bar{W}^{\lambda}=-\frac{1}{\gamma} J \lambda .
$$

This combined with (3.92) yields the value (3.20) of the symmetric matrix $M$. 
Proof of Proposition 3.8. Let $v=\left(v_{1}, v_{2}\right) \in W^{1, r}(\Omega)^{2}$. Considering the functions $v_{\varepsilon}^{i}, i=1,2$, which are defined by (3.68) and satisfy the convergences (3.76), we have

$$
\begin{aligned}
E_{\varepsilon}:= & \int_{\Omega}\left|D u_{\varepsilon}-D u-v_{1} D v_{\varepsilon}^{1}-v_{2} D v_{\varepsilon}^{2}\right|^{2} d x \\
= & \int_{\Omega}\left|D u_{\varepsilon}\right|^{2} d x-\int_{\Omega}|D u|^{2} d x+\int_{\Omega}\left(v_{1}^{2}\left|D v_{\varepsilon}^{1}\right|^{2}+v_{2}^{2}\left|D v_{\varepsilon}^{2}\right|^{2}\right) d x \\
& -2 \int_{\Omega}\left(v_{1} D u_{\varepsilon}: D v_{\varepsilon}^{1}+v_{2} D u_{\varepsilon}: D v_{\varepsilon}^{2}\right) d x+o(1) .
\end{aligned}
$$

Putting $u_{\varepsilon}$ in equation (3.57) and $u$ in equation (3.61) we get

$$
\begin{aligned}
\int_{\Omega}\left|D u_{\varepsilon}\right|^{2} d x-\int_{\Omega}|D u|^{2} d x & =\langle f, u\rangle_{H^{-1}(\Omega)^{2}, H_{0}^{1}(\Omega)^{2}}-\int_{\Omega}|D u|^{2} d x+o(1) \\
& =\int_{\Omega} \Gamma u \cdot u d x+o(1) \\
& =\frac{\gamma}{4\left(\gamma^{2}+1\right)} \int_{\Omega}|u|^{2} d x+o(1) .
\end{aligned}
$$

Moreover, putting $V_{\varepsilon}^{i}$ in estimate (3.72) together with $V_{\varepsilon}^{i}=e_{i}$ in $Q_{r_{\varepsilon}}$, (3.71), (3.73), (3.56), and using the $\varepsilon Y$-periodicity of $D v_{\varepsilon}^{i}$, we get

$$
\left|D v_{\varepsilon}^{i}\right|^{2} \longrightarrow \lim _{\varepsilon \rightarrow 0}\left(\frac{1}{\varepsilon^{2}} f_{Y}\left|D V_{\varepsilon}^{i}\right|^{2} d y\right)=\frac{\gamma}{4} \quad \text { weakly-* in } \mathcal{M}(\bar{\Omega}),
$$

hence since $v_{i} \in C(\bar{\Omega})$,

$$
\int_{\Omega}\left(v_{1}^{2}\left|D v_{\varepsilon}^{1}\right|^{2}+v_{2}^{2}\left|D v_{\varepsilon}^{2}\right|^{2}\right) d x=\frac{\gamma}{4} \int_{\Omega}|v|^{2} d x+o(1) .
$$

Estimates (3.96), (3.97) and (3.98) thus imply that

$$
\begin{aligned}
E_{\varepsilon}= & \frac{\gamma}{4\left(\gamma^{2}+1\right)} \int_{\Omega}|u|^{2} d x+\frac{\gamma}{4} \int_{\Omega}|v|^{2} d x \\
& -2 \int_{\Omega}\left(v_{1} D u_{\varepsilon}: D v_{\varepsilon}^{1}+v_{2} D u_{\varepsilon}: D v_{\varepsilon}^{2}\right) d x+o(1) .
\end{aligned}
$$

On the other hand, applying the estimate (3.75) with the function $v=v_{i} u_{\varepsilon}, i=$ 1,2 , and using the convergences (3.76), (3.73), (3.56) and (3.79), we obtain

$$
\begin{aligned}
\int_{\Omega} D v_{\varepsilon}^{i}: D u_{\varepsilon} v_{i} d x & =\int_{\Omega} D v_{\varepsilon}^{i}: D\left(v_{i} u_{\varepsilon}\right) d x+o(1) \\
& =\frac{\gamma_{\varepsilon}^{i}}{\varepsilon^{2}} e_{i} \cdot\left(\int_{\Omega} \frac{1_{\omega_{\varepsilon}}}{\left|Q_{r_{\varepsilon}}\right|} u_{\varepsilon} v_{i} d x-\int_{\Omega} \frac{1_{Y \backslash Q}}{|Y \backslash Q|}\left(\frac{x}{\varepsilon}\right) u_{\varepsilon} v_{i} d x\right)+o(1) \\
& =\frac{\gamma^{2}}{4\left(\gamma^{2}+1\right)} \int_{\Omega} e_{i} \cdot(\gamma I-J) u v_{i} d x-\frac{\gamma}{4} \int_{\Omega} u_{i} v_{i} d x+o(1) .
\end{aligned}
$$


This combined with (3.99) yields

$$
\begin{aligned}
E_{\varepsilon}= & \frac{\gamma}{4\left(\gamma^{2}+1\right)} \int_{\Omega}|u|^{2} d x+\frac{\gamma}{4} \int_{\Omega}|v|^{2} d x \\
& +\frac{\gamma}{2\left(\gamma^{2}+1\right)} \int_{\Omega} u \cdot v d x+\frac{\gamma^{2}}{2\left(\gamma^{2}+1\right)} \int_{\Omega} J u \cdot v d x+o(1) .
\end{aligned}
$$

Putting the function

$$
v:=-\frac{1}{\gamma^{2}+1}(I+\gamma J) u
$$

in (3.100) we get

$$
E_{\varepsilon}=\int_{\Omega}\left|D u_{\varepsilon}-D u-v_{1} D v_{\varepsilon}^{1}-v_{2} D v_{\varepsilon}^{2}\right|^{2} d x \underset{\varepsilon \rightarrow 0}{\longrightarrow} 0
$$

Finally, since the sequences $v_{\varepsilon}^{i}$ strongly converge to zero in $L^{\frac{2 r}{r-2}}(\Omega)^{2}$ by (3.76) and $u \in W^{1, r}(\Omega)^{2}$, the strong convergence (3.65) is a straightforward consequence of (3.101).

\section{References}

[1] S. AlinhaC and P. GÉRARD, "Pseudo-differential Operators and the Nash-Moser Theorem", translated from the 1991 French original by Stephen S. Wilson, Graduate Studies in Mathematics, Vol. 82, American Mathematical Society, Providence, RI, 2007.

[2] G. Allaire, Homogenization of the Navier-Stokes equations in open sets perforated with tiny holes I, Abstract framework, a volume distribution of holes, Arch. Rational Mech. Anal., 113 (1991), 209-259.

[3] M.E. BogovsKi, Solution of the of the first boundary value problem for the equation of continuity of an incompressible medium, Soviet Math. Dokl. 20 (1979), 1094-1098.

[4] M. BRIANE, Homogenization of the Stokes equations with high-contrast viscosity, J. Math. Pures Appl. 82 (7) (2003), 843-876.

[5] H.C. BRINKMAN, A calculation of the viscous force exerted by a flowing fluid on a dense swarm of particles, Appl. Sci. Res. A1 (1947), 27-34.

[6] D. CiORANESCU and F. Murat, Un terme étrange venu d'ailleurs, I \& II, In: "Nonlinear Partial Differential Equations and their Applications, Collège de France Seminar", II and III, H. Brezis and J.-L. Lions (eds.), Research Notes in Math., Vol. 60 and 70, Pitman, London, 1982, 98-138 and 154-178. English translation: A strange term coming from nowhere, In: "Topics in the Mathematical Modelling of Composite Materials", A. Cherkaev and R. V. Kohn. (eds.), Progress in Nonlinear Differential Equations and their Applications, Vol. 31, Birkhäuser, Boston, 1997, 44-93.

[7] G. Dal Maso and A. Garroni, New results on the asymptotic behaviour of Dirichlet problems in perforated domains, Math. Models Methods Appl. Sci. 3 (1994), 373-407.

[8] P. GÉRARD, Micro-local defect measures, Comm. Partial Differential Equations 16 (1991), 1761-1794.

[9] G. Geymonat, Sul problema di Dirichlet per le equazioni lineari ellittiche, Ann. Scuola Norm. Sup. Pisa Cl. Sci. 16 (1962), 225-284. 
[10] O. A. Ladyzhenskaya, "The Mathematical Theory of Viscous Incompressible Flow", translated from the Russian, Mathematics and its Applications, Vol. 2, Gordon and Breach, Science Publishers, New York-London-Paris, 1969.

[11] F. Murat, Compacité par compensation, Ann. Scuola Norm. Sup. Pisa Cl. Sci. 5 (1978), 489-507.

[12] C. PIDERI and P. SEPPECHER, A second gradient material resulting from the homogenization of an heterogeneous linear elastic medium, Contin. Mech. Thermodyn. 9 (1997), 241-257.

[13] E. Sanchez-Palencia, "Non Homogeneous Materials and Vibration Theory", Monographs in Physics, Vol. 127, Springer-Verlag, Berlin, 1980.

[14] L. TARTAR, Homogénéisation en hydrodynamique, In: "Singular Perturbation and Boundary Layer Theory", Lecture Notes in Mathematics, Vol. 597, Springer, Berlin-Heidelberg, 1977, 474-481.

[15] L. TARTAR, Remarks on homogenization, In: "Homogenization and Effective Moduli of Materials and Media", IMA Vol. Math. Appl., Vol. 1, Springer, New-York 1986, 228-246.

[16] L. TARTAR, H-measures, a new approach for studying homogenisation, oscillations and concentration effects in partial differential equations, Proc. Roy. Soc. Edinburgh Sect. A 115A (1990), 193-230.

[17] L. TARTAR, "The General Theory of Homogenization: A Personalized Introduction", Lecture Notes of the Unione Matematica Italiana, Springer-Verlag, Berlin Heidelberg, 2009.

[18] L. TARTAR, “Topics in Nonlinear Analysis", Publications Mathématiques d'Orsay, Vol. 78 , Orsay 1978.

INSA de Rennes

IRMAR, CNRS UMR 6625

35708 Rennes Cedex 7, France

mbriane@insa-rennes.fr

Université Paris-Sud

LMO, CNRS UMR 8628

91405 Orsay, France

Institut Universitaire de France

Patrick.Gerard@math.u-psud.fr 